\title{
Seeds Of Life In Space (SOLIS): The Organic Composition Diversity at 300-1000 au Scale in Solar-type Star-forming Regions*
}

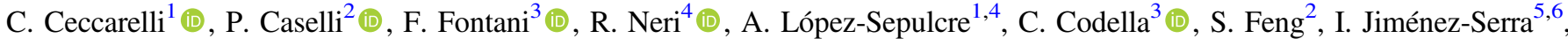

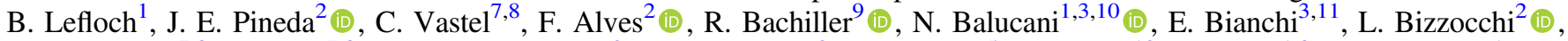 \\ S. Bottinelli ${ }^{7,8}$, E. Caux ${ }^{7,8}$, A. Chacón-Tanarro ${ }^{2}$, R. Choudhury ${ }^{2}$, A. Coutens ${ }^{5}$, F. Dulieu ${ }^{12}$, C. Favre ${ }^{1,3}$, P. Hily-Blant ${ }^{1}$ (1), \\ J. Holdship ${ }^{5}$ (D) , C. Kahane ${ }^{1}$, A. Jaber Al-Edhari, ${ }^{1,13}$, J. Laas ${ }^{2}$ (D) , J. Ospina ${ }^{1}$, Y. Oya ${ }^{14}$, L. Podio ${ }^{3}$, A. Pon ${ }^{2,15}$ (D) A. Punanova ${ }^{2}$ (D), \\ D. Quenard ${ }^{5,6}$, A. Rimola $^{16}$ (D), N. Sakai ${ }^{17}$ (D) I. R. Sims ${ }^{18}$, S. Spezzano ${ }^{2}$, V. Taquet ${ }^{3}$ (D) , L. Testi ${ }^{3,19}$ (D), P. Theulé ${ }^{20}$, P. Ugliengo ${ }^{21}$, \\ A. I. Vasyunin ${ }^{2,22}$ (D), S. Viti ${ }^{5}$ (D) L. Wiesenfeld ${ }^{1}$, and S. Yamamoto ${ }^{14}$ \\ ${ }^{1}$ Université Grenoble Alpes, CNRS, IPAG, F-38000 Grenoble, France; cecilia.ceccarelli@ univ-grenoble-alpes.fr \\ ${ }^{2}$ Max-Planck-Institut für extraterrestrische Physik, Giessenbachstrasse 1, D-85748 Garching, Germany; caselli@mpe.mpg.de \\ ${ }^{3}$ INAF-Osservatorio Astrofisico di Arcetri, Largo E. Fermi 5, I-50125, Florence, Italy \\ ${ }^{4}$ Institut de Radioastronomie Millimétrique, 300 Rue de la Piscine, F-38406, Saint-Martin d'Hères, France \\ ${ }^{5}$ Department of Physics and Astronomy, University College London, Gower Street, London, WC1E 6BT, UK \\ ${ }^{6}$ School of Physics and Astronomy, Queen Mary University of London, 327 Mile End Road, London, E1 4NS, UK \\ ${ }^{7}$ Université de Toulouse, UPS-OMP, IRAP, Toulouse, France \\ ${ }^{8}$ CNRS, IRAP, 9 Av. Colonel Roche, BP 44346, F-31028 Toulouse Cedex 4, France \\ ${ }^{9}$ Observatorio Astronómico Nacional (OAN, IGN), Calle Alfonso XII, 3, E-28014 Madrid, Spain \\ ${ }^{10}$ Dipartimento di Chimica, Biologia e Biotecnologie, Università di Perugia, Via Elce di Sotto 8, I-06123 Perugia, Italy \\ ${ }^{11}$ Dipartimento di Fisica e Astronomia, Universitá degli Studi di Firenze, Italy \\ ${ }^{12}$ LERMA, Université de Cergy-Pontoise, F-95000 Cergy Pontoise Cedex, France \\ ${ }^{13}$ University of AL-Muthanna, College of Science, Physics Department, AL-Muthanna, Iraq \\ ${ }^{14}$ Department of Physics, The University of Tokyo, Bunkyo-ku, Tokyo 113-0033, Japan \\ ${ }^{15}$ Department of Physics and Astronomy, The University of Western Ontario, 1151 Richmond Street, London, N6A 3K7, Canada \\ ${ }^{17}$ Departament de Química, Universitat Autònoma de Barcelona, E-08193 Bellaterra, Spain
The Institute of Physical and Chemical Research (RIKEN), 2-1, Hirosawa, Wako-shi, Saitama 351-0198, Japan \\ ${ }^{18}$ Institut de Physique de Rennes, UMR CNRS 6251, Université de Rennes 1, 263 Avenue du Général Leclerc, F-35042 Rennes Cedex, France \\ ${ }^{19}$ European Southern Observatory, Karl-Schwarzschild-Str. 2, D-85748 Garching bei München, Germany \\ ${ }^{20}$ Aix-Marseille Université, PIIM UMR-CNRS 7345, F-13397 Marseille, France \\ ${ }^{21}$ Dipartimento di Chimica and NIS Centre, Università degli Studi di Torino, Via P. Giuria 7, I-10125 Torino, Italy \\ ${ }^{22}$ Ural Federal University, Ekaterinburg, Russia \\ Received 2017 July 23; revised 2017 October 20; accepted 2017 October 23; published 2017 November 30
}

\begin{abstract}
Complex organic molecules have been observed for decades in the interstellar medium. Some of them might be considered as small bricks of the macromolecules at the base of terrestrial life. It is hence particularly important to understand organic chemistry in Solar-like star-forming regions. In this article, we present a new observational project: Seeds Of Life In Space (SOLIS). This is a Large Project using the IRAM-NOEMA interferometer, and its scope is to image the emission of several crucial organic molecules in a sample of Solar-like star-forming regions in different evolutionary stages and environments. Here we report the first SOLIS results, obtained from analyzing the spectra of different regions of the Class 0 source NGC 1333-IRAS4A, the protocluster OMC-2 FIR4, and the shock site L1157-B1. The different regions were identified based on the images of formamide $\left(\mathrm{NH}_{2} \mathrm{CHO}\right)$ and cyanodiacetylene $\left(\mathrm{HC}_{5} \mathrm{~N}\right)$ lines. We discuss the observed large diversity in the molecular and organic content, both on large (3000-10,000 au) and relatively small (300-1000 au) scales. Finally, we derive upper limits to the methoxy fractional abundance in the three observed regions of the same order of magnitude of that measured in a few cold prestellar objects, namely $\sim 10^{-12}-10^{-11}$ with respect to $\mathrm{H}_{2}$ molecules.
\end{abstract}

Key words: ISM: abundances - ISM: clouds - ISM: molecules - radio lines: ISM

\section{Introduction}

From a simple chemical point of view, all terrestrial living organisms, from microbes to humans, are made up of the same basic components: amino acids, fatty acids, sugars, nucleobases, and so on. In total, we are referring to about 50 small molecules containing less than 100 atoms of carbon, with hydrogen, oxygen, nitrogen, and other elements in smaller quantities; terrestrial life is based on organic chemistry. Of course, this is not by chance, but due to the electronic structure of $\mathrm{C}$ atoms and their abundance (not locked in refractory/rocky

\footnotetext{
* Based on observations carried out under project number L15AA with the IRAM-NOEMA interferometer. IRAM is supported by INSU/CNRS (France), MPG (Germany), and IGN (Spain).
}

material). Thus it does not come as a surprise that the largest molecules detected in the interstellar medium (ISM), and those having more than six atoms, contain carbon (http://www.astro. uni-koeln.de/cdms/molecules; Müller et al. 2001). Moreover, species like formamide $\left(\mathrm{NH}_{2} \mathrm{CHO}\right)$, believed to be a crucial molecule in the synthesis of metabolic and genetic species in modern versions of the Urey-Miller experiment (Saladino et al. 2012), are easily found in regions forming Solar-like stars (Kahane et al. 2013; Mendoza et al. 2014; López-Sepulcre et al. 2015) as well as in external galaxies (Müller et al. 2013). One step further, amino acids are found in cometary and meteoritic material (Pizzarello et al. 1991; Elsila et al. 2009; Altwegg et al. 2016). This evidence led the Nobel laureate C. De Duve to affirm that the chemical seeds of life are universal 
Table 1

List of the iCOMs Detected in Solar-like Star-forming Regions

\begin{tabular}{lll}
\hline \hline Molecule Name & Formula & References \\
\hline Methanol & $\mathrm{CH}_{3} \mathrm{OH}$ & 1,2 \\
Methanethiol & $\mathrm{CH}_{3} \mathrm{SH}$ & 3 \\
Methyl cyanide & $\mathrm{CH}_{3} \mathrm{CN}$ & 4 \\
Formamide & $\mathrm{NH}_{2} \mathrm{CHO}$ & 5 \\
Propyne & $\mathrm{CH}_{3} \mathrm{CCH}$ & 6,7 \\
Ethylene oxide & $\mathrm{C}_{2} \mathrm{H}_{4} \mathrm{O}$ & 8 \\
Acetaldehyde & $\mathrm{CH}_{3} \mathrm{CHO}$ & $6,9,10$ \\
Methyl isocyanate & $\mathrm{CH}_{3} \mathrm{NCO}$ & 11,12 \\
Cyanoacetylene & $\mathrm{HC}_{5} \mathrm{~N}$ & 13 \\
Methyl formate & $\mathrm{HCOOCH}_{3}$ & $14,15,16$ \\
Glycol aldehyde & $\left.\mathrm{HCO}_{(} \mathrm{CH}_{2}\right) \mathrm{OH}$ & $17,18,19$ \\
Acetic acid & $\mathrm{CH}_{3} \mathrm{COOH}$ & 20 \\
Dimethyl ether & $\mathrm{CH}_{3} \mathrm{OCH}$ & $14,13,16$ \\
Ethanol & $\mathrm{CH}_{3} \mathrm{CH}_{2} \mathrm{OH}$ & $21,18,16$ \\
Ethyl cyanide & $\mathrm{CH}_{3} \mathrm{CH}_{2} \mathrm{CN}$ & $14,15,22$ \\
Acetone & $\mathrm{CH}_{3} \mathrm{COCH}_{3}$ & 8 \\
Propanal & $\mathrm{CH}_{3} \mathrm{CH}_{2} \mathrm{CHO}$ & 8 \\
Ethylene glycol & $\left(\mathrm{CH}_{2} \mathrm{OH}\right)_{2}$ & 17 \\
\hline
\end{tabular}

Note. Last column provides representative relevant references.

References. 1. Bizzocchi et al. (2014), 2. Maret et al. (2005), 3. Majumdar et al. (2016), 4. van Dishoeck et al. (1995), 5. López-Sepulcre et al. (2015), 6. Vastel et al. (2014), 7. Caux et al. (2011), 8. Lykke et al. (2017), 9. Jaber et al. (2014), 10. Codella et al. (2015), 11. Ligterink et al. (2017), 12. MartinDoménech et al. (2017), 13. Jaber Al-Edhari et al. (2017), 14. Jiménez-Serra et al. (2016), 15. Cazaux et al. (2003), 16. Lefloch et al. (2017a), 17. Jørgensen et al. (2012), 18. Taquet et al. (2015), 19. Coutens et al. (2015), 20. Jørgensen et al. (2016), 21. Bisschop et al. (2008), 22. López-Sepulcre et al. (2017).

and life is an obligatory manifestation of matter, written into the fabric of the universe (De Duve 2005, 2011).

One of the remarkable discoveries of modern astronomy is that relatively complex organic molecules are present in the ISM (e.g., Herbst \& van Dishoeck 2009). In the following discussion, we will refer to C-bearing molecules containing at least six atoms as iCOMs, for "interstellar complex organic molecules." ${ }^{23}$ They may be seen as the smallest bricks from which the larger "chemical seeds of life" mentioned by De Duve are formed. Although iCOMs have been observed for decades in massive star formation regions (e.g., Rubin et al. 1971), the detection of these special molecules in regions that will eventually form Solar-like planetary systems came much later (Ceccarelli et al. 2000b; Cazaux et al. 2003). This was a crucial discovery that set a direct link between organic chemistry in the ISM and in the solar system, and provided additional and crucial ground to the "universal chemical seeds of life" hypothesis.

Yet despite the great importance of iCOMs, their routes of formation and destruction are still largely debated (e.g., Herbst \& van Dishoeck 2009; Caselli \& Ceccarelli 2012, Vasyunin \& Herbst 2013; Balucani et al. 2015; Enrique-Romero et al. 2016; Vasyunin et al. 2017). The reasons for this impasse are multiple. One of them is the lack of systematic observational studies that would put strong constraints on theory, when observations are compared to models. In particular, the vast

\footnotetext{
23 Please note that we added "i" to the commonly used COMs acronym in order to be clear that these molecules are only complex in the interstellar context, contrary to what chemists would consider complex in the terrestrial context.
}

majority of the 43 so far detected iCOMs are only observed toward SgrB2 (e.g., Belloche et al. 2017). In Solar-like starforming regions, only 18 iCOMs have been detected so far (Table 1). Probably even more important for building up a theory, most of these iCOMs are observed in very few sources. The most studied ones are the hot corino sources, of which the prototype and best studied one is IRAS16293-2422 (e.g., van Dishoeck et al. 1995; Ceccarelli et al. 2000a; Jaber et al. 2014; Jørgensen et al. 2016). iCOMs are present in large quantities also in the shocks created by the outflows from Solar-like protostars, but also here studies exist only toward a very limited number of sources (e.g., Requena-Torres et al. 2007; Arce et al. 2008; Codella et al. 2015; Lefloch et al. 2017a). Interestingly, iCOMs are present in the lukewarm clouds of the Galactic Center (Requena-Torres et al. 2006), likely due to the widespread shocks in the region. Finally and surprisingly, iCOMs have also been detected in very few of the coldest known sources, the prestellar cores (Öberg et al. 2010; Bacmann et al. 2012; Cernicharo et al. 2012; Vastel et al. 2014; Jiménez-Serra et al. 2016). In summary, the observational framework is too sparse to provide strong constraints to theory.

In this respect, the case of methyl formate (MF) dimethyl ether (DME) provides an illustrative example of why observations toward a larger number of objects and with a better spatial resolution are needed to firmly establish how these species are formed. The available observations, cited previously, show that MF and DME are present in hot and cold environments, and that their abundances are correlated (e.g., Jaber et al. 2014). Early models claimed that both species are synthesized on the grain surfaces, thanks to reactions between radicals created by the UV illumination of the grain mantles during the cold ( $\leqslant 20 \mathrm{~K}$ ) phase (e.g., Garrod et al. 2008) and the so-called warm-up phase models. However, radicals become mobile only at dust temperatures larger than about $25-30 \mathrm{~K}$, so that in cold environments the process would be inhibited. In addition, the liberation of MF and DME from the grain surfaces at low temperature would be problematic (e.g., Minissale et al. 2016). Alternatively, it has been suggested that MF and DME are synthesized in the gas phase from the methanol, formed on the grain surface by the hydrogenation of $\mathrm{CO}$ and UV photodesorbed (e.g., Balucani et al. 2015). Spatially resolved maps of the two species, plus that of methanol, in different regions where the thermal structure is well-known and with a different UV radiation field would allow one to distinguish which process dominates in what environment.

In order to attack this problem, we started a Large Program at the NOEMA interferometer called Seeds Of Life In Space (SOLIS). The immediate goal of SOLIS is to provide a homogeneous data set of observations of five crucial iCOMs in half a dozen targets representative of Solar-like systems in their first evolutionary stages. The ultimate goal is to have a more complete view of how organic chemistry starts and develops during the first stages of evolution of Solar-like systems. The present article presents the overall SOLIS project and some results based on the first observations around $82 \mathrm{GHz}$, with a moderate spatial resolution. A series of accompanying articles provides additional and detailed analysis of single SOLIS targets: L1157-B1 by Codella et al. (2017) and S. Feng et al. (2017, in preparation); OMC-2 FIR4 by Fontani et al. (2017), Favre et al. (2017), and R. Neri et al. (2017, in preparation); IRAS4A by A. López-Sepulcre et al. (2017, in preparation); 
Table 2

List of the SOLIS Targets

\begin{tabular}{|c|c|c|c|c|c|c|c|c|}
\hline Source & Type & $\begin{array}{l}\text { Lum } \\
\left(L_{\odot}\right)\end{array}$ & $\begin{array}{l}\text { Dist } \\
(\mathrm{pc})\end{array}$ & R.A. & Decl. & $V_{\mathrm{LSR}}$ & ASAI & CHESS \\
\hline L1544 & prestellar core & $\cdots$ & 140 & $05: 04: 12.2$ & $25: 10: 42.8$ & 7.3 & $\mathrm{Y}$ & $\mathrm{Y}$ \\
\hline $\mathrm{L} 1521 \mathrm{~F}$ & VeLLO & 0.1 & 140 & $04: 28: 39.0$ & $26: 51: 35.6$ & 6.4 & $\mathrm{~N}$ & $\mathrm{~N}$ \\
\hline NGC 1333-IRAS4A & Class 0 & 10 & 260 & $03: 29: 10.5$ & $31: 13: 30.9$ & 6.5 & $\mathrm{Y}$ & $\mathrm{Y}$ \\
\hline CepE & Class 0 & 100 & 730 & 23:03:13.0 & 61:42:21.0 & -10.9 & $\mathrm{Y}$ & $\mathrm{N}$ \\
\hline NGC 1333-SVS13A & Class I & 34 & 260 & 03:29:03.7 & $31: 16: 03.8$ & 8.5 & Y & $\mathrm{N}$ \\
\hline OMC-2 FIR4 & protocluster & $\leqslant 1000$ & 420 & $05: 35: 27.0$ & $-05: 09: 56.8$ & 11.4 & Y & $\mathrm{Y}$ \\
\hline
\end{tabular}

Note. The different columns report the name of each source, type (see text for more details), luminosity, distance, coordinates of the center of the observations, and local standard of rest velocity. The references to the source type, luminosity, and distance are reported in the text. The last two columns report whether each source is a target of the ASAI and CHESS Large Programs (Ceccarelli et al. 2010; Lefloch et al. 2017b).

L1544 by Punanova et al. (2017). Forthcoming articles will present the remaining observations obtained, also with higher spatial resolution.

This article is organized as follows. We present the targeted iCOMs and their transitions in Section 2, together with the targeted sources and the motivations for the choices. In Section 3 we present the results of the first observations, obtained with the same frequency setting, that permits a first analysis of the similarity and differences among the observed sources, as discussed in Section 4. A final section, Section 5, concludes the article.

\section{Project Presentation}

The goal of the SOLIS project is to build a homogeneous data set of observations from which we can constrain the routes of formation and destruction of a selected number of iCOMs during the earliest phases of formation of a Solar-like system. To this end, we selected a sample of sources representative of these first phases, described in Section 2.1, and obtained high spatial resolution images of selected lines of a sample of molecules, described in Section 2.2, crucial to understand the major routes of formation and destruction of iCOMs.

\subsection{Source Sample}

SOLIS aims to cover the first evolutionary phases of the Solar-type star formation process, by observing several sources, even though the sample will inevitably be incomplete. The sources were selected based on the results of two previous Large Programs: Chemical Herschel Surveys of Star-forming Regions (CHESS; Ceccarelli et al. 2010) and ASAI (Astrochemical Surveys at Iram; Lefloch et al. 2017b). Both programs consist of single-dish unbiased spectral surveys of representative star-forming regions: CHESS covers the $500-1900 \mathrm{GHz}$ band with Herschel/HIFI, whereas ASAI covers the 3, 2, and $1.3 \mathrm{~mm}$ bands observable with the IRAM-30 $\mathrm{m}$ telescope. The SOLIS selected sources are listed in Table 2. They are all wellknown sources representative of different evolutionary phases: the prestellar core L1544, the VeLLO (Very Low Luminosity Object) L1521F, the low mass Class 0 source NGC 1333IRAS4A, the intermediate mass Class 0 source CepE, the low mass Class I source NGC 1333-SVS13A, the protocluster OMC-2 FIR4, and the molecular shock L1157-B1. In the following we provide a brief description of each source.

\subsection{1. $L 1544$}

L1544 is a prestellar core in the Taurus complex, at a distance of 140 pc (Kenyon et al. 1994; Schlafly et al. 2014). This prestellar core has been studied extensively, and its chemical and physical structure is well-known. The gas temperature reaches a minimum of $6 \mathrm{~K}$ toward the central 2000 au and a maximum of $13 \mathrm{~K}$ at 15,000 au (Crapsi et al. 2007). Caselli et al. (1999) measured large amounts of CO freeze-out within the central few 1000 au, and within the COdepleted zone, deuterated molecules thrive (Caselli et al. 2002, 2003; Bacmann et al. 2003; Vastel et al. 2006; Crapsi et al. 2007). The ground state transition of ortho- $\mathrm{H}_{2} \mathrm{O}$ reveals an inverse P-Cygni profile that can be reproduced with a simple chemical-dynamical model describing the quasi-equilibrium contraction of a Bonnor-Ebert sphere (Caselli et al. 2012; Keto et al. 2015; Quenard et al. 2016). L1544 is hence a prestellar core on the verge of star formation. Thus its study will provide us with crucial information about the chemical processes before the switch-on of the protostar. Several iCOMs are detected toward this core (Bizzocchi et al. 2014; Vastel et al. 2014; Jiménez-Serra et al. 2016). NOEMA observations are centered on the position of the $\mathrm{CH}_{3} \mathrm{OH}$ peak (Bizzocchi et al. 2014), where iCOMs are also expected to peak (Vastel et al. 2014; Jiménez-Serra et al. 2016), about 5000 au away from the dust peak, as confirmed by the theoretical work of Vasyunin et al. (2017).

\subsection{2. $L 1521 F$}

$\mathrm{L} 1521 \mathrm{~F}$ is another dense core in the Taurus molecular cloud. It was studied by Crapsi et al. (2004), who found a striking similarity with L1544, with the exception of a factor of 2 lower deuterium fraction and a peculiar velocity field. Based on these results, Crapsi et al. (2004) defined L1521F as "a highly evolved starless core." In fact, two years later, a faint infrared source was discovered at its dust peak by the Spitzer Space Telescope (Bourke et al. 2006). This source belongs to the group of VeLLOs (i.e., objects with a luminosity lower than about $0.1 L_{\odot}$ ) embedded in dense cores (Kauffmann et al. 2005; Dunham et al. 2006). More recently, the central regions of L1521F were studied with ALMA: Tokuda et al. (2014) imaged dust continuum emission and simple molecules $\left(\mathrm{HCO}^{+}, \mathrm{HCN}, \mathrm{CS}\right)$, finding large central densities and substructure with an arc-like morphology. This structure resembles the hydrodynamical simulations of infalling material around protostars carried out by Matsumoto et al. (2015). Tokuda et al. (2016) studied in detail the structure of the L1521F core, from 
10 s to $\sim 10,000 \mathrm{au}$, revealing an inner region with a flat density structure where an unresolved protostellar source is next to a starless high-density core. In summary, L1521F represents the next evolutionary stage of a prestellar core such as L1544.

\subsubsection{NGC 1333-IRAS4A}

NGC 1333-IRAS4A is located in the Perseus NGC 1333 region at a distance of $(260 \pm 26)$ pc (Hirota et al. 2008; Schlafly et al. 2014). It was the second discovered hot corino (Bottinelli et al. 2004)-namely hot $(\geqslant 100 \mathrm{~K})$, dense $\left(\geqslant 10^{7} \mathrm{~cm}^{-3}\right)$, and compact $(\leqslant 100 \mathrm{au}$ in radius) regions enriched of iCOMs (Ceccarelli et al. 2007; Caselli \& Ceccarelli 2012). Interferometric observations showed that it is a binary system composed of two Class 0 objects separated by $\sim 1$ !" 8 (423 au): IRAS4-A1 and IRAS4-A2 (e.g., Looney et al. 2000; Santangelo et al. 2015; Tobin et al. 2016). The overall bolometric luminosity of IRAS4A and its total envelope mass are estimated to be $9.1 L_{\odot}$ and 5.6 $M_{\odot}$, respectively (Kristensen et al. 2012; Karska et al. 2013; Tobin et al. 2016). While IRAS4A1 is more than three times brighter at $1.3 \mathrm{~mm}$ than its companion, only IRAS4-A2 is associated with a hot corino (Coutens et al. 2015; Taquet et al. 2015; De Simone et al. 2017; López-Sepulcre et al. 2017). In addition, IRAS4-A1 drives a fast collimated jet associated with bright $\mathrm{H}_{2}$ emission, whereas IRAS4-A2 powers a slower and precessing jet (Santangelo et al. 2015). These findings suggest that the two Class 0 sources are likely in different evolutionary stages, although it is not possible so far to firmly conclude whether this is due to evolution or rather accretion luminosity effects/bursts (De Simone et al. 2017; López-Sepulcre et al. 2017). The compact jets from IRAS4-A1 and IRAS4-A2 create a shock site $\sim 12^{\prime \prime}$ south, where SOLIS observations show emission from iCOMs, for the first time (see Section 3.2).

\subsubsection{CepE}

CepE is located in the second most massive molecular cloud of the Cepheus OB3 association at a distance of $730 \mathrm{pc}$ (Sargent 1977; Few \& Cohen 1983). It is an isolated Class 0 source of intermediate mass associated with the infrared source IRAS $23011+6126$. Its luminosity is $\sim 100 L_{\odot}$ and its mass is $35 M_{\odot}$ (Lefloch et al. 1996; Crimier et al. 2009). CepE drives an exceptionally powerful outflow, the southern lobe of which is terminated by the Herbig-Haro object HH377 (Lefloch et al. 1996, 2015). Studies of the millimeter CO and the near-infrared $\mathrm{H}_{2}$ and [Fe II] lines revealed the presence of several outflows (Eislöffel et al. 1996; Ladd \& Hodapp 1997). Continuum and line observations obtained with the IRAM-PdBI (Plateau de Bure Interferometer) at $1^{\prime \prime}$ scale confirm the presence of a binary protostellar system, with each component driving a high-velocity jet detected in lines of $\mathrm{H}_{2}, \mathrm{CO}$, and $\mathrm{SiO}$ (J. Ospina-Zamudio et al. 2017, in preparation). The dynamical timescale of these ejections is short-typically 1000 years. The same observations also show evidence for the presence of a hot corino in one of the two components, so that this is a good target to understand the influence of luminosity on the formation and destruction of iCOMs.

\subsubsection{NGC 1333-SVS13A}

NGC 1333-SVS13A is located in the Perseus NGC 1333 region at a distance of $260 \pm 26$ pc (Hirota et al. 2008; Schlafly et al. 2014), close to the other SOLIS source NGC 1333-IRAS4A. SVS13A is part of a small cluster of protostars, of which the two brightest sources, labelled A and B, are separated by $\sim 15^{\prime \prime}$ (e.g., Chini et al. 1997; Bachiller et al. 1998; Looney et al. 2000; Chen et al. 2009; Tobin et al. 2016). SVS13A is relatively evolved, as it possesses a ratio $L_{\text {submm }} / L_{\text {bol }} \sim 0.08$, typical of Class I sources. Its luminosity is $\sim 33 L_{\odot}$ (Tobin et al. 2016). SVS13A drives the famous chain of Herbig-Haro $(\mathrm{HH})$ objects 7-11 (Reipurth et al. 1993) and is associated with an extended outflow (>0.07 pc: Lefloch et al. 1998; Codella et al. 1999). For comparison, SVS13B is a Class 0 protostar with $L_{\text {bol }} \sim 1 L_{\odot}$ (Tobin et al. 2016; De Simone et al. 2017) that drives a well-collimated $\mathrm{SiO}$ jet (Bachiller et al. 1998). Recent observations revealed the presence of a compact $(\sim 50 \mathrm{au})$, hot $(\sim 200 \mathrm{~K})$, and dense $\left.\geqslant 10^{7} \mathrm{~cm}^{-3}\right)$ region in SVS13A, hinting at the presence of a hot corino region, the first ever detected in a Class I source (Codella et al. 2016; De Simone et al. 2017). More recently, Bianchi et al. (2017) measured a deuterium fraction in SVS13A smaller than in Solarlike prestellar cores and Class 0 sources (their Figure 9), showing, for the first time, that there may be a chemical evolution between Class 0 and I sources. Thus SVS13A is a perfect target to understand the evolutionary effects in the iCOMs abundancesnamely formation and destruction routes.

\subsubsection{OMC-2 FIR4}

OMC-2 FIR4 belongs to the Orion Molecular Complex, at a distance of 420 pc (Hirota et al. 2007; Schlafly et al. 2014). The total FIR4 mass and luminosity are around $30 M_{\odot}$ (Mezger et al. 1990; Crimier et al. 2009) and less than $1000 L_{\odot}$ (Crimier et al. 2009; Furlan et al. 2014), respectively. Interferometric observations showed that FIR4 contains several protostars and clumps, even though their number is uncertain (Shimajiri et al. 2008; López-Sepulcre et al. 2013; Gonzalez-Garcia et al. 2016). Our new SOLIS maps detect at least eight sources, of which only one has a FIR counterpart so that the others are, likely, very young Class 0 or prestellar objects (R. Neri et al. 2017, in preparation). Therefore, FIR4 is certainly a young protocluster. The nature of the hosted objects is practically unknown, including their mass, temperature, chemical composition, and dynamical status. However, what makes this protocluster particularly interesting and the reason why it is a SOLIS target is that FIR4 is, at present, the best known analogue of the Sun progenitor for two reasons. The first one is that the Sun was born in a cluster of stars and not in an isolated clump (Adams 2010). The second reason is that the solar system experienced a large irradiation of energetic $(\geqslant 10 \mathrm{MeV})$ particles during its first phases of formation (Gounelle et al. 2006). A similar dose of energetic particles is present somewhere in or close to FIR4, producing an enhanced degree of gas ionization (Ceccarelli et al. 2014; Fontani et al. 2017). Finally, close to FIR4 lie two additional sources, FIR3 and FIR5, about 25" north and south, respectively. Not much was known about these two sources before our SOLIS observations. Shimajiri et al. (2015) obtained an unbiased spectral survey at 3 and $0.8 \mathrm{~mm}$ toward FIR3, with single-dish telescopes and corresponding angular resolution of about $20^{\prime \prime}$. Based on those observations, Shimajiri et al. claim that the observed line emission is dominated by the shocked gas from the outflow rising from FIR3 and hitting FIR4 rather than a hot core/corino sources. 
Table 3

Molecules Targeted by the SOLIS Observations

\begin{tabular}{lcccl}
\hline \hline \multicolumn{1}{c}{ Molecule } & $E_{\text {up }}(\mathrm{K})$ & Lines & Role in Chemistry \\
\hline Methoxy & $\mathrm{CH}_{3} \mathrm{O}$ & $4-83$ & 5 & $\begin{array}{l}\text { Key radical, precursor of dimethyl ether and other iCOMs. Predicted to form either via the reaction } \mathrm{CH}_{3} \mathrm{OH}+ \\
\text { OH in the gas (measured rate), or via photolysis of frozen } \mathrm{CH}_{3} \mathrm{OH} \text { on the grain mantles (rate not measured/ } \\
\text { known). }\end{array}$ \\
\hline Methanol & $\mathrm{CH}_{3} \mathrm{OH}$ & $7-30$ & 6 & $\begin{array}{l}\text { A mother molecule, gas phase precursor of several iCOMs. } \\
\text { Formed on the grain surfaces by hydrogenation of frozen } \mathrm{CO} \text { (measured } \\
\end{array}$ \\
& & & $\begin{array}{l}\text { rate but debated). Not clear how it is released in the gas phase in cold gas, } \\
\text { possibly by cosmic rays or reactive desorption, but with uncertain rates. }\end{array}$ \\
\hline
\end{tabular}

Dimethyl ether $\mathrm{CH}_{3} \mathrm{OCH}_{3} \quad 19-150 \geqslant 30 \quad$ Predicted to form on the grain surfaces via UV/particle irradiation and/or

(DME) from radicals on the surfaces, or in the gas phase by the reaction of $\mathrm{CH}_{3} \mathrm{O}$ and $\mathrm{CH}_{3}$. Its detection in cold environments is difficult to explain within our current understanding of grain-surface radical chemistry, as radicals cannot diffuse at low temperatures. The release from the grains into the gas is problematic as laboratory experiments show that it would rather break the molecule. The rate of the gas-phase radiative association reaction is highly uncertain; that on the grain surfaces is unknown.

\begin{tabular}{lllll}
\hline $\begin{array}{l}\text { Methyl formate } \\
\text { (MF) }\end{array}$ & $\mathrm{HCOOCH}_{3}$ & $30-550 \geqslant 20$ & $\begin{array}{l}\text { Predicted to form either on the grain surfaces by reaction of } \mathrm{CH}_{3} \mathrm{O} \text { with } \mathrm{HCO} \\
\text { and } \mathrm{O} \text { (rate known), or in the gas phase from a sequence of reactions starting } \\
\text { from DME (measured rates). As for DME, the grain-surface formation route is } \\
\text { challenged by the MF detection in cold environments (see text). }\end{array}$ \\
\hline Formamide & $\mathrm{NH}_{2} \mathrm{HCO}$ & $4-83$ & 4 & $\begin{array}{l}\text { Initially predicted to form on the grain surfaces by hydrogenation of } \\
\text { frozen } \mathrm{HNCO} \text { laboratory experiments do not confirm this route. Recent } \\
\text { theoretical computations predict formation via the gas-phase reaction of } \\
\mathrm{NH}_{2} \text { and } \mathrm{H}_{2} \mathrm{CO} .\end{array}$ \\
\hline
\end{tabular}

Note. They will be observed with the high spectral resolution provided by the narrow band correlator (see Table 4). The third and fourth columns report the upper level energy range of the lines in the WideX correlator and their number. The last column lists a very brief summary of the formation of each molecule. This is not meant to be exhaustive, but just to give a general overview. Specific SOLIS articles will address the issue in more depth.

\subsection{7. $L 1157-B 1$}

This famous shocked region is associated with the Class 0 protostar L1157-mm, at a distance of $250 \mathrm{pc}$ (Looney et al. 2007). L1157-mm has a luminosity of $\simeq 3 L_{\odot}$ (Tobin et al. 2010), and drives an episodic and precessing jet (Gueth et al. 1996, 1998; Podio et al. 2016). In the last $\leqslant 2000 \mathrm{yr}$, the jet accelerated and entrained material, creating a spectacular bipolar outflow and opening cavities along the path. The south and blueshifted lobe of the outflow is formed by two cavities, the respective apexes of which are known as B1 and B2 (e.g., Gueth et al. 1998). The latter is more distant from L1157-mm and, hence, older than B1. The SOLIS target is B1, the age of which is estimated to be less than 2000 years (Podio et al. 2016). This low age and the extent of the shock allow us to probe chemical composition variations along the shock and, consequently, to test the chemistry where the dust icy mantles are recently released in the gas phase via sputtering. In the context of the SOLIS goal, B1 provides us with the possibility to test the different theories of iCOMs formation. Indeed, the different ejection episodes caused several shocks where the jet impacted the cavities walls. In these sites, the abundance of a large number of species is dramatically enhanced: from diatomic, such as $\mathrm{SiO}$ and $\mathrm{CS}$, to more complex molecules, such as $\mathrm{CH}_{3} \mathrm{CN}$ and $\mathrm{CH}_{3} \mathrm{CHO}$ (see, e.g., Bachiller et al. 2001; Benedettini et al. 2013; Codella et al. 2015). Lately, several iCOMs were detected in B1 with abundances as large as or more than in hot corinos (Lefloch et al. 2017a). However, just a few iCOMs (methanol, methyl cyanide, and acetaldehyde) have been imaged with interferometers so far (Codella et al. 2007, 2015; Fontani et al.
2014). These images show a inhomogeneous distribution of the molecules, associated with the different ejection episodes. The comparison of the distribution of acetaldehyde and formamide obtained with the new SOLIS observations provides us with very stringent constraints on the formation route of formamide (Codella et al. 2017).

\subsection{Target Species and Lines}

In the zoo of the detected iCOMs, we selected five species to observe with high spectral resolution, as summarized in Table 3. These species were chosen because they are known to be abundant in warm $(\geqslant 50 \mathrm{~K})$ and, sometimes, cold $(\leqslant 20 \mathrm{~K})$ environments, and have the potential to discriminate between the principal chemical mechanisms at work-specifically, (i) grain surface versus gas phase chemistry, (ii) the exact formation and destruction routes (e.g., asserting whether a class of reactions, such as gas radiative associations or surface photolysis, plays an important role), and (iii) the desorption yield and efficiency. Table 3 provides a very short summary of what is known or hypothesized about their formation routes.

To give a practical example, two major routes of formation of formamide are discussed in the literature: (1) on the grain surfaces, either via reactions occurring between radicals when the grain temperature allows them to be mobile (at $\geqslant 30 \mathrm{~K}$; e.g., Garrod \& Herbst 2006; Garrod et al. 2008) or thanks to UV/ particle ice irradiation (Bennett \& Kaiser 2007; Jones et al. 2011; Fedoseev et al. 2016; Kanuchova et al. 2016); and (2) in the gas phase from the reaction of formaldehyde $\left(\mathrm{H}_{2} \mathrm{CO}\right)$ and amidogen $\left(\mathrm{NH}_{2}\right)$ (Kahane et al. 2013; Barone et al. 2015; Vazart et al. 2016; Skouteris et al. 2017). Formaldehyde and 
Table 4

List of the Frequency Setups of SOLIS

\begin{tabular}{|c|c|c|c|c|c|c|c|}
\hline \multirow{2}{*}{ Setup } & \multicolumn{2}{|c|}{ Spat.Res. } & \multicolumn{2}{|c|}{ Frequency Range $(\mathrm{GHz})$} & \multirow{2}{*}{$\begin{array}{l}\text { Vel.Res. } \\
\left(\mathrm{km} \mathrm{s}^{-1}\right)\end{array}$} & \multirow{2}{*}{ Species } & \multirow{2}{*}{$\begin{array}{c}\mathrm{rms} \\
\left(\mathrm{mJy} \mathrm{beam}^{-1}\right)\end{array}$} \\
\hline & $(")$ & (au) & WideX & Narrow Band & & & \\
\hline $1^{\mathrm{a}, \mathrm{b}}$ & $\sim 4$ & $300-1000$ & $80.80-84.40$ & $81.60-82.60$ & 0.57 & Methoxy, Formamide & $4-5$ \\
\hline $2^{\mathrm{c}}$ & $\sim 4$ & $300-1000$ & $80.80-84.40$ & $81.60-82.60$ & 0.14 & Methoxy, Formamide & $8-9$ \\
\hline $3^{\mathrm{a}}$ & $\sim 4$ & $300-1000$ & $95.85-99.45$ & $96.65-97.65$ & 0.48 & Methanol, Dimethyl ether, Methyl formate & $4-5$ \\
\hline $4^{\mathrm{c}}$ & $\sim 4$ & $80-200$ & $95.85-99.45$ & $96.65-97.65$ & 0.12 & Methanol, Dimethyl ether, Methyl formate & $8-9$ \\
\hline $5^{\mathrm{a}}$ & $\sim 1$ & $80-200$ & 204.0-207.6 & $204.8-205.8$ & 0.91 & Methanol, Dimethyl ether, Methyl formate & $7-14$ \\
\hline
\end{tabular}

Notes.The columns report the setup number, the spatial resolution in arcsec and au, the frequency range in the WideX and narrow band correlator, the velocity resolution of the latter, the species in Table 3 targeted with the narrow band correlator, and the reached rms. Please note that the present article only reports observations obtained with setup 1 .

a This setup is only used for the "hot" sources, namely NGC 1333-IRAS4A, CepE, NGC 1333-SVS13A, OMC-2 FIR4, and L1157-B1.

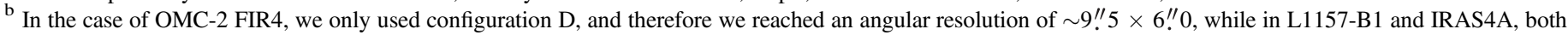
configurations $\mathrm{C}$ and $\mathrm{D}$ were used and we reached an angular resolution of $\sim 3$ !" $5-4$ ".

c This setup is only used for the "cold" sources, namely L1544 and L1521F.

amidogen are the products of hydrogenation on the grain surfaces of $\mathrm{CO}$ and $\mathrm{N}$, respectively, occurring during the cold prestellar phase (e.g., Vasyunin et al. 2017). The SOLIS observations of the distribution of the formamide abundance compared with other iCOMs can distinguish between the two theories, as they have different formation and destruction timescales, as we show in Codella et al. (2017).

More generally, abundance maps of iCOMs in different environments, cold and warm, also help find which theory is the correct one and to constrain the underlying chemistry, including the desorption mechanism in different zones: interstellar UV photons versus photons generated by cosmic ray impacts with $\mathrm{H}_{2}$ molecules, and chemical reactive and thermal desorption. This analysis will be facilitated by the comparison with laboratory measurements and theoretical computations (also) carried out by members of the SOLIS team (see, for example, Minissale et al. 2016 and Skouteris et al. 2017).

The observations of the species listed in Table 3 are obtained via five setups, summarized in Table 4 . In addition to the five molecules in Table 3, the NOEMA WideX large band correlator provides us with several other lines and species, including more iCOMs, which are a precious complement to further constrain the models.

\section{Observations and Results}

\subsection{Observations and Analysis}

We report here the first set of observations carried out toward three SOLIS targets: L1157-B1, OMC-2 FIR4, and NGC 1333IRAS4A. The observations took place during the summerwinter 2015 in the setup 1 of Table 4 . We used the array in configurations $\mathrm{D}$ and $\mathrm{C}$, with baselines from $\sim 15$ to $\sim 240 \mathrm{~m}$, providing angular resolutions going from $\sim 9$ ". $5 \times 6$ ". 0 for the D configuration only (used for OMC-2 FIR4) to $\sim 4$ ". $0-3$ ". 5 for the combined C $+\mathrm{D}$ configurations (used for L1157-B1 and NGC 1333-IRAS4A). The phase centers and local standard of rest velocities were set to the values listed in Table 2 . The primary beam is $\sim 61^{\prime \prime}$. The system temperature was between 100 and $200 \mathrm{~K}$ in almost all tracks, and the amount of precipitable water vapor was generally around $10 \mathrm{~mm}$. The calibration of the bandpass and of the absolute flux scale were performed on the usual NOEMA calibrators, 3C454.3 and MWC349 (when available). The calibration of the gains in phase and amplitude was performed on strong quasars close to each source (angular distance $\leqslant 20^{\circ}$ ). For details on the calibrators used for each individual source, please see the specific articles listed in the introduction.

We used both the Narrow Band and the WideX correlators, which provide us data with different bandwidths and spectral resolutions (see Table 4). The continuum of each source was imaged by averaging the line-free channels of the WideX correlator units. Calibration and imaging were performed using the CLIC and MAPPING softwares of the GILDAS ${ }^{24}$ package using standard procedures. For OMC-2 FIR4 and NGC $1333-$ IRAS4A, for which a strong continuum was detected, the continuum image was self-calibrated (in phase and amplitude), and the solutions were applied to the lines. This could not be performed for L1157-B1, for which the continuum was not detected. The analysis of the spectral lines was performed using standard procedures of the CLASS software, which is part of the GILDAS package mentioned previously. All of the spectral parameters used to interpret the observations are retrieved from the Cologne Database for Molecular Spectroscopy (CDMS; Müller et al. 2001, 2005).

\subsection{Results}

Figure 1 shows the maps of the three imaged sources: OMC-2 FIR4, NGC 1333-IRAS4A, and L1157-B1. In each map, we marked the regions that posses the spectra analyzed as follows. Please note that specific articles (see the introduction) are devoted to each single source. Our goal here is to show the importance of having interferometric observations to disentangle and reveal the chemical similarities and differences inside each region within a source and among the different sources at various scales.

L1 157-B1: We identified three regions, based on the analysis presented in Codella et al. (2017): SHOCK 1, 2, and 3. Briefly, based on the different abundance distribution of acetaldehyde and formamide, the region SHOCK 1 contains the gas most recently shocked by the jet impinging on the B1 cavity wall, whereas the region SHOCK 3 marks the oldest shocked gas in $\mathrm{B} 1$, with a difference in time of $\leqslant 1000$ years (see also Podio et al. 2016).

\footnotetext{
${ }^{24}$ The GILDAS software is developed at the IRAM and the Observatoire de Grenoble, and is available at http://www.iram.fr/IRAMFR/GILDAS.
} 

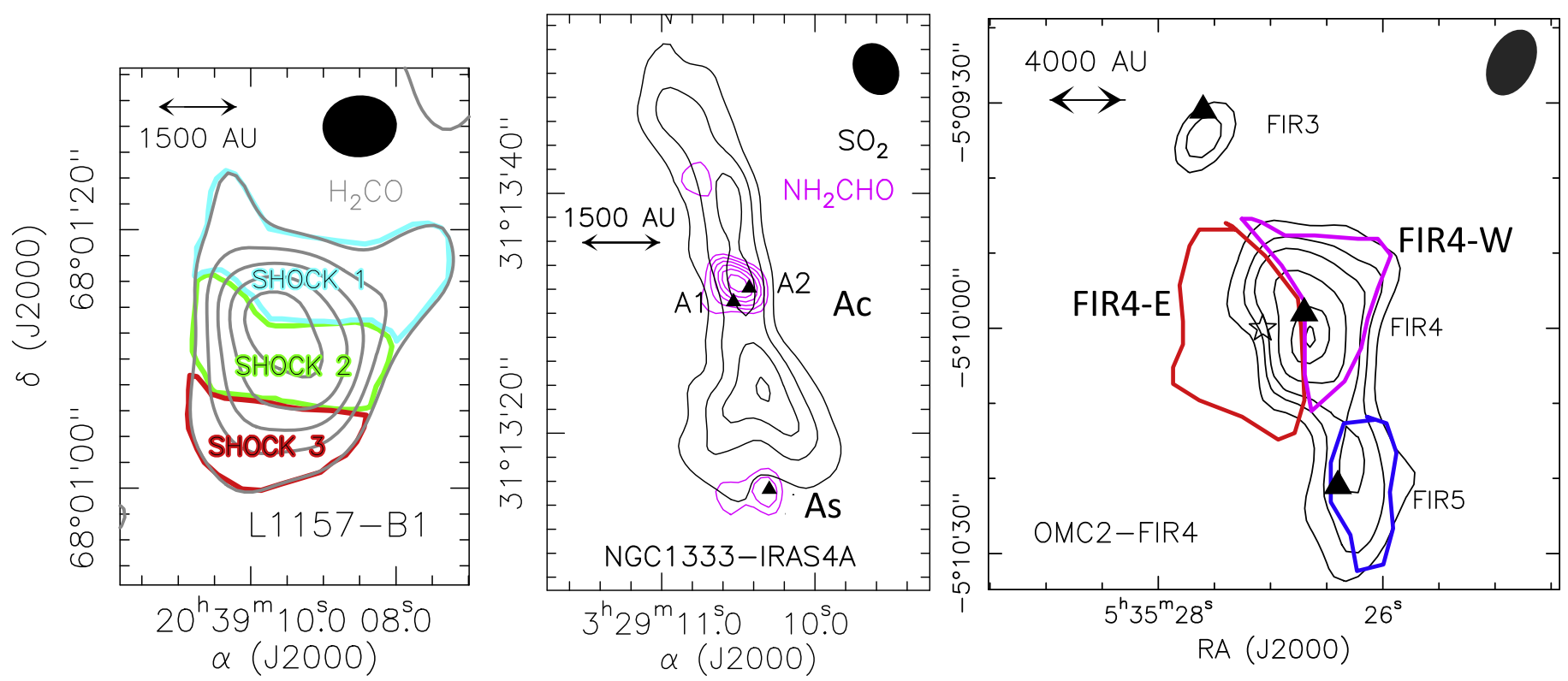

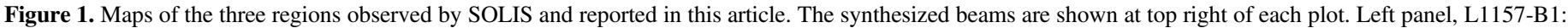

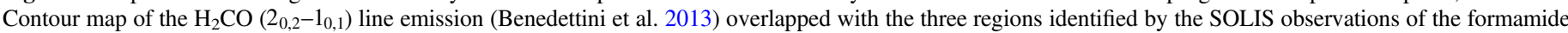

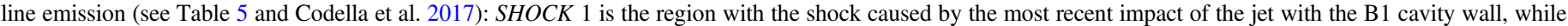

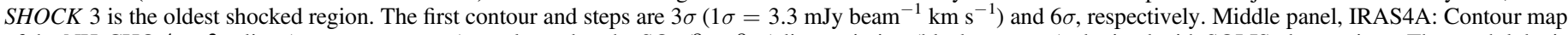

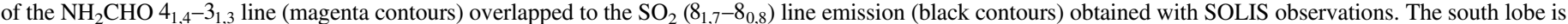

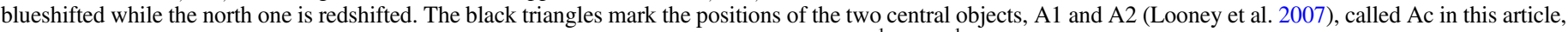

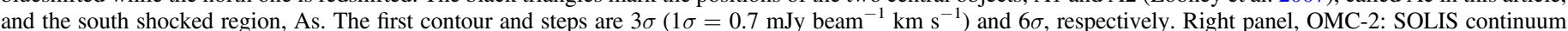

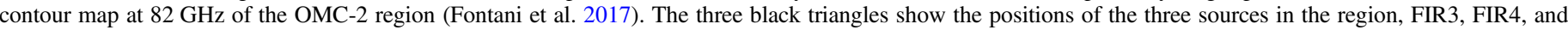

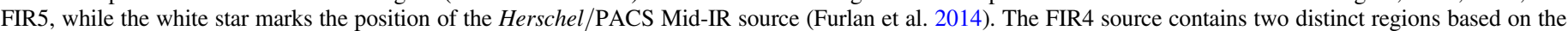

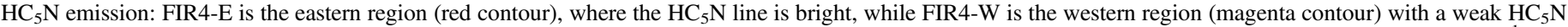

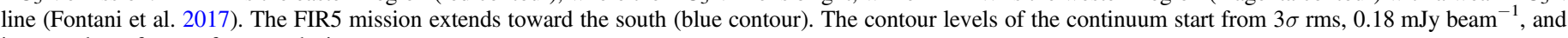
increase by a factor of two each time.

OMC-2 FIR4: The NOEMA primary beam encompasses three sources: FIR3, FIR4, and FIR5. In Fontani et al. (2017), we show that FIR4 consists of two regions, west and east, marked in the following FIR4-W and FIR4-E, respectively. These two regions differ in the $\mathrm{HC}_{3} \mathrm{~N}(9-8) / \mathrm{HC}_{5} \mathrm{~N}(31-30)$ line emission ratio. Considering the possible difference in the excitation conditions, the different line emission ratio translates into a different $\mathrm{HC}_{3} \mathrm{~N} / \mathrm{HC}_{5} \mathrm{~N}$ abundance ratio and/or gas temperature of the two regions.

NGC 1333-IRAS4A: We identified two regions. The first one, marked Ac, is coincident with the well-known hot corino IRAS4A, which consists of two objects, A1 and A2, not resolved by the present observations (e.g., Taquet et al. 2015; López-Sepulcre et al. 2017). The second region, marked as As, is southeast of the hot corino. It presents weak, but definitively detected, formamide line emission. Note that in As, as in L1157-B1, no continuum is detected, so that it is very likely a shock site. Supporting this hypothesis, the formamide emission is blueshifted as other tracers of the two jets emanating from $\mathrm{A} 1$ and $\mathrm{A} 2$, and the emission region coincides with the apex of the $\mathrm{SO}_{2}$ emission that probes the jets (Santangelo et al. 2015).

The WideX spectra obtained by integrating the emission over the previously noted regions are shown in Figure 2. A zoom-in around the $81.5 \mathrm{GHz}$ frequency range, which is particularly rich in lines, is shown in Figure 3 . Table 5 summarizes the detected lines in each source/region and the measured integrated fluxes. Note that we consider a detection when the line integrated flux is above $5 \sigma$.

Finally, for comparison, in Figure 4 we show the IRAM$30 \mathrm{~m}$ spectra obtained toward the three sources in the same frequency range of Figure 2. At these frequencies, the main beam of IRAM-30 $\mathrm{m}$ is $\sim 30^{\prime \prime}$, namely about 3 to 10 times larger than the NOEMA synthesized beams. Note that the IRAM-30 m spectra were obtained within the Large Project $\mathrm{ASAI}^{25}$ described in Lefloch et al. (2017b).

\section{Discussion}

We organize this discussion into three parts. The first part discusses the overall similarities and diversity among the three sources and the regions composing them, while the second part discusses the three sources separately. A third section discusses the detection and non-detection of the two SOLIS target molecules of setup 1: formamide and methoxy.

\subsection{Overall Sources Similarity and Diversity}

\subsubsection{Large (3000-10,000 au) Scale Line Emission}

The comparison between the SOLIS (Figure 2) and ASAI (Figure 4) spectra provides straightforward information on the emission at the 3000-10,000 au scales probed by the ASAI observations and filtered out by the SOLIS ones. For example, the CCS line is very bright in the ASAI spectra, and weak (L1157-B1 SHOCK 1 and 2, and FIR4-E), if not absent (IRAS4Ac and FIR5), in the SOLIS ones, a clear indication that CCS is abundant in the extended molecular clouds to which L1157-B1, OMC-2 FIR4, and NGC 1333-IRAS4A belong. The same can be said of the $\mathrm{H}_{2} \mathrm{CCO}, \mathrm{c}-\mathrm{C}_{3} \mathrm{H}_{2}$, and $\mathrm{HC}_{5} \mathrm{~N}$ lines, which are prominent in the ASAI spectra and weak or absent in the SOLIS ones. All these species are small

\footnotetext{
${ }^{25}$ Astrochemical Surveys at IRAM: http://www.oan.es/asai/.
} 


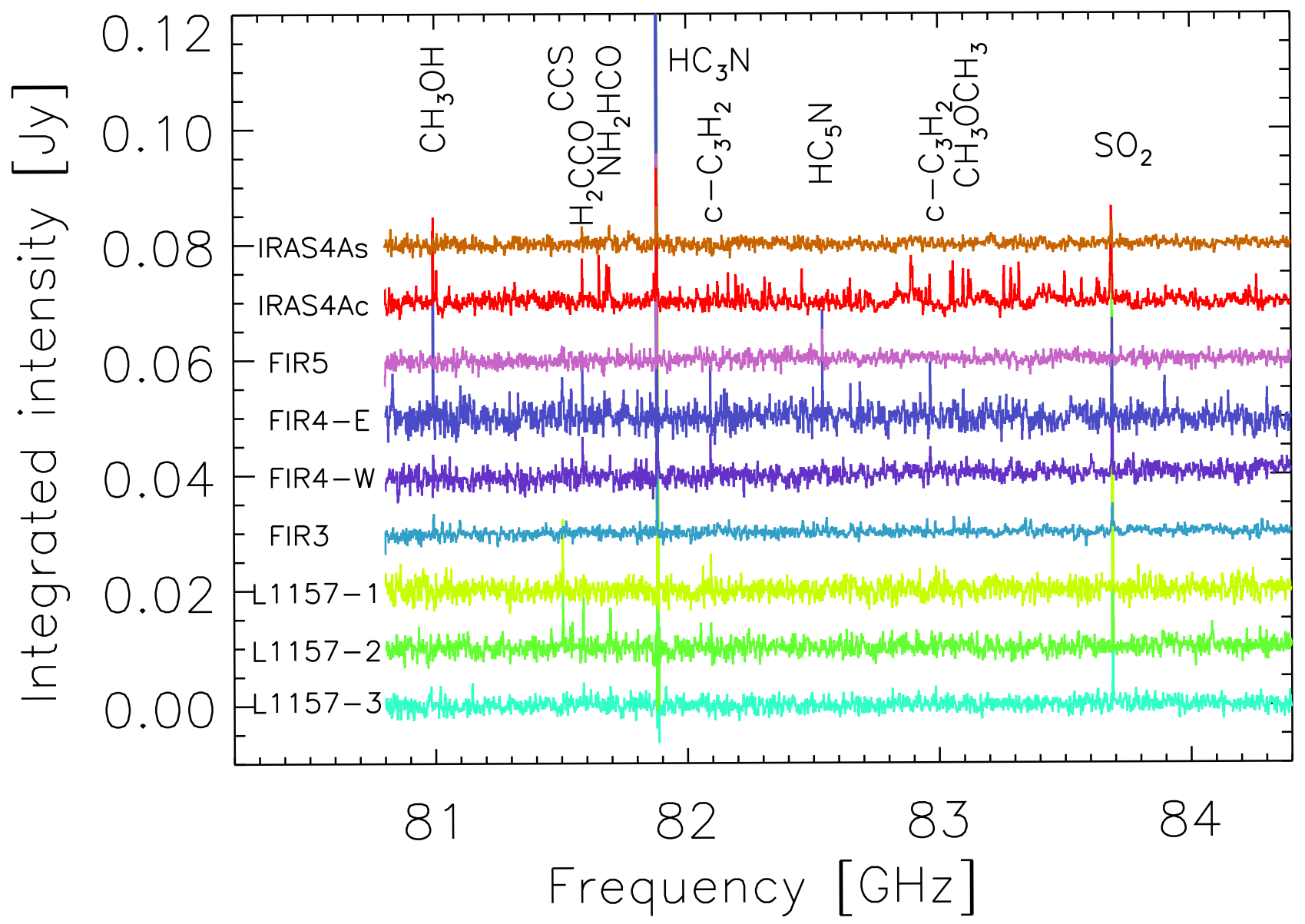

Figure 2. WideX spectra of the eight regions described in the text and marked in Figure 1, from the bottom: L1157-3 = L1157-B1 SHOCK 3, L1157-2 = L1157-B1 SHOCK 2, L1157-1 = L1157-B1 SHOCK 1, FIR3 = OMC-2 FIR3, FIR4-W = OMC-2 FIR4 west, FIR4-E = OMC-2 FIR4 east, FIR5 = OMC-2 FIR5, IRAS4Ac = IRAS4A hot corino, and IRAS4As = IRAS4A shocked region. The integrated intensity is in Jy and the frequency in GHz. An arbitrary offset is added to each spectrum. The brightest detected lines are labeled at the top of the plot.

hydrocarbons or unsaturated C-bearing chains particularly abundant in the skin of the parental clouds (e.g., Spezzano et al. 2017). Two dedicated SOLIS articles discuss the details regarding the cyanopolyynes (Fontani et al. 2017) and c- $\mathrm{C}_{3} \mathrm{H}_{2}$ (Favre et al. 2017) toward OMC-2 FIR4.

Note that the ASAI spectra are relatively similar in OMC-2 FIR4 and NGC 1333-IRAS4A, with the biggest exception being the $\mathrm{HC}_{5} \mathrm{~N}$ line, totally absent in NGC 1333-IRAS4A and very bright in OMC-2 FIR4. In Fontani et al. (2017), we argue that this is due to the anomalously large energetic $(\geqslant 10 \mathrm{MeV})$ particle irradiation of the OMC-2 FIR4 region, first revealed by Herschel observations (Ceccarelli et al. 2014). On the other hand, the similarity suggests the presence of an embedded hot corino in OMC-2 FIR4.

The shocked region L1157-B1 shows a relatively different line spectrum with respect to both OMC-2 FIR4 and NGC 1333-IRAS4A, with bright lines of formamide (Codella et al. 2017) and $\mathrm{SO}_{2}$ (S. Feng et al. 2017, in preparation). This difference with respect to OMC-2 FIR4 is less surprising, as the single-dish ASAI spectrum in the latter is likely dominated by a dense and cold gas highly irradiated by energetic particles (Ceccarelli et al. 2014; Fontani et al. 2017); namely it is likely due to the very different environment of OMC-2 FIR4 and L1157-B1. A bit more surprising is the difference between L1157-B1 and NGC 1333-IRAS4, as in both sources the emission presumably (also) originates from species directly or indirectly injected by the grain mantles. This difference is probably due to either a different chemical timescale or a different initial grain mantle composition.

\subsubsection{Chemical Diversity at the 300-1000 au Scale}

The sources and the regions surrounding them appear particularly different at the 300-1000 au scale. The spectra of Figures 2 and 3 clearly identify either different zones of line emission or different chemical composition across each target source.

All the $80.8-84.3 \mathrm{GHz}$ spectra are dominated by the $\mathrm{HC}_{3} \mathrm{~N}$ (9-8) line and, to a lesser extent, the $\mathrm{SO}_{2}\left(8_{1,7}-8_{0,8}\right)$ line (see Table 6). Yet the relative intensity with respect to other lines varies from region to region. In particular, the $\mathrm{HC}_{3} \mathrm{~N}(9-8)$ to $\mathrm{SO}_{2}\left(8_{1,7}-8_{0,8}\right)$ intensity ratio varies from about unity in NGC 1333-IRAS4As to 20 in OMC-2 FIR4-E. Such a large difference is unlikely due to excitation effects, as the two lines have rather similar upper level energies, so it probably reflects a real chemical difference. Figure 5 shows the predicted line ratio as a function of the excitation temperature, assuming LTE and optically thin lines. The figure shows that this ratio varies by a factor of about four between 6 and $100 \mathrm{~K}$. Therefore, NGC 1333-IRAS4As appears to be a region enriched in $\mathrm{SO}_{2}$ compared with OMC-2 FIR4-E or, alternatively, poorer in $\mathrm{HC}_{3} \mathrm{~N}$. L1157-B1 lies in between, with a $\mathrm{HC}_{3} \mathrm{~N}$ (9-8) to $\mathrm{SO}_{2}$ 


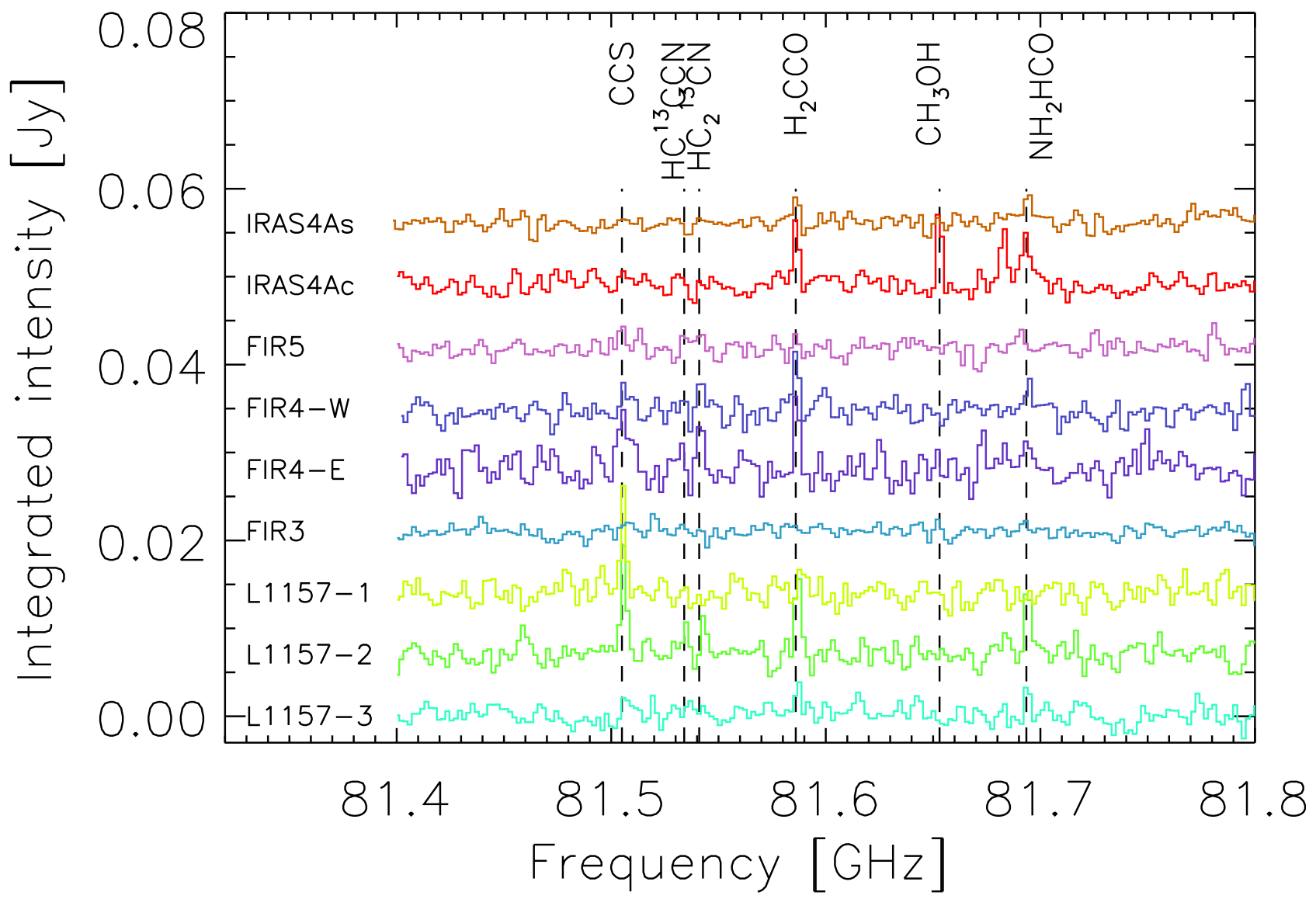

Figure 3. SOLIS setup 1 WideX spectra of the eight regions described in the text and marked in the figure, following the notation of Figure 2 and Table 5. The integrated intensity is in Jy and the frequency in GHz. The brightest detected lines are labeled at the top of the plot.

Table 5

List of Lines Detected by SOLIS in the 80.80-84.40 GHz Frequency Range (Setup 1 of Table 4) toward the Regions NGC 1333-IRAS4A (IRAS4A in the header), the OMC-2 FIR4 (OMC-2 in the header), and L1157-B1 ${ }^{\mathrm{a}}$

\begin{tabular}{|c|c|c|c|c|c|c|c|c|c|c|c|c|c|}
\hline \multirow{2}{*}{ Species } & \multirow{2}{*}{ Transition } & \multirow{2}{*}{$\begin{array}{c}\nu^{\mathrm{b}} \\
(\mathrm{GHz})\end{array}$} & \multirow{2}{*}{$\begin{array}{c}E_{\mathrm{u}}^{\mathrm{b}} \\
(\mathrm{K})\end{array}$} & \multirow{2}{*}{$\log \left(A_{\mathrm{ij}} / \mathrm{s}^{-1}\right)^{\mathrm{b}}$} & \multicolumn{2}{|c|}{${\text { IRAS } 4 A^{c}}^{c}$} & \multicolumn{4}{|c|}{ OMC $-2^{c}$} & \multicolumn{3}{|c|}{$\mathrm{L} 1157-\mathrm{B} 1^{\mathrm{c}}$} \\
\hline & & & & & As & $\overline{A c}$ & FIR5 & FIR4-W & FIR4-E & FIR3 & 1 & 2 & 3 \\
\hline $\mathrm{CH}_{3} \mathrm{OH}$ & $7_{2,6}-8_{1,7} \mathrm{~A}$ & 80.99324 & 103 & -5.98 & $\mathrm{~N}$ & Y & $\mathrm{N}$ & $\mathrm{N}$ & $\mathrm{Y}$ & $\mathrm{N}$ & $\mathrm{N}$ & $\mathrm{N}$ & $\mathrm{N}$ \\
\hline $\mathrm{CCS}$ & $6_{7}-5_{6}$ & 81.50517 & 15 & -4.61 & $\mathrm{~N}$ & $\mathrm{~N}$ & $\mathrm{~N}$ & $\mathrm{~N}$ & Y & $\mathrm{N}$ & $\mathrm{Y}$ & $\mathrm{Y}$ & $\mathrm{N}$ \\
\hline $\mathrm{HC}^{13} \mathrm{CCN}$ & $9-8$ & 81.53411 & 20 & -4.38 & $\mathrm{~N}$ & $\mathrm{~N}$ & $\mathrm{~N}$ & $\mathrm{~N}$ & $\mathrm{Y}$ & $\mathrm{N}$ & $\mathrm{N}$ & $\mathrm{Y}$ & $\mathrm{N}$ \\
\hline $\mathrm{HCC}^{13} \mathrm{CN}$ & $9-8)$ & 81.54198 & 20 & -4.38 & $\mathrm{~N}$ & $\mathrm{~N}$ & $\mathrm{~N}$ & $\mathrm{~N}$ & $\mathrm{Y}$ & $\mathrm{N}$ & $\mathrm{N}$ & $\mathrm{Y}$ & $\mathrm{N}$ \\
\hline $\mathrm{H}_{2} \mathrm{CCO}$ & $4_{1,3}-3_{1,2}$ & 81.58623 & 23 & -5.27 & $\mathrm{Y}$ & Y & $\mathrm{N}$ & $\mathrm{N}$ & Y & $\mathrm{N}$ & $\mathrm{N}$ & $\mathrm{Y}$ & $\mathrm{N}$ \\
\hline $\mathrm{NH}_{2} \mathrm{CHO}$ & $4_{1,4}-3_{1,3}$ & 81.69345 & 13 & -4.43 & Y & $\mathrm{Y}$ & $\mathrm{N}$ & $\mathrm{Y}$ & Y & $\mathrm{N}$ & $\mathrm{N}$ & $\mathrm{Y}$ & $\mathrm{Y}$ \\
\hline $\mathrm{HC}_{3} \mathrm{~N}$ & 9-8 & 81.88147 & 20 & -4.38 & $\mathrm{Y}$ & Y & $\mathrm{Y}$ & $\mathrm{Y}$ & Y & Y & $\mathrm{Y}$ & $\mathrm{Y}$ & $\mathrm{Y}$ \\
\hline$c-\mathrm{C}_{3} \mathrm{H}_{2}$ & $2_{0,2}-1_{1,1}$ & 82.09354 & 6 & -4.72 & $\mathrm{~N}$ & $\mathrm{~N}$ & $\mathrm{~N}$ & $\mathrm{Y}$ & Y & $\mathrm{N}$ & $\mathrm{Y}$ & $\mathrm{Y}$ & $\mathrm{N}$ \\
\hline $\mathrm{HC}_{5} \mathrm{~N}$ & $31-30$ & 82.53904 & 63 & -4.21 & $\mathrm{~N}$ & $\mathrm{~N}$ & $\mathrm{Y}$ & $\mathrm{N}$ & Y & $\mathrm{N}$ & $\mathrm{N}$ & $\mathrm{N}$ & $\mathrm{N}$ \\
\hline$c-\mathrm{C}_{3} \mathrm{H}_{2}$ & $3_{1,2}-3_{0,3}$ & 82.96620 & 16 & -5.00 & $\mathrm{~N}$ & Y & $\mathrm{N}$ & $\mathrm{Y}$ & $\mathrm{Y}$ & $\mathrm{N}$ & $\mathrm{N}$ & $\mathrm{N}$ & $\mathrm{N}$ \\
\hline $\mathrm{SO}_{2}$ & $8_{1,7}-8_{0,8}$ & 83.68809 & 37 & -5.17 & $\mathrm{Y}$ & $\mathrm{Y}$ & $\mathrm{N}$ & $\mathrm{Y}$ & $\mathrm{Y}$ & $\mathrm{Y}$ & $\mathrm{Y}$ & $\mathrm{Y}$ & $\mathrm{Y}$ \\
\hline
\end{tabular}

Notes. "Y" means that the line is detected"; "N" means that the line is undetected. Additional lines are detected toward NGC 1333-IRAS4A, but we report in this table only the lines detected in at least two sources.

${ }^{a}$ As reported in the text, lines are considered detected when the flux peak is at least 5 times the rms noise.

b The Cologne Database for Molecular Spectroscopy (CDMS;http://www.astro.uni-koeln.de/cdms/; Müller et al. 2001, 2005) molecular database was used for retrieving the spectroscopic data obtained by Xu and Lovas (1997), Saito et al. (1987), Lovas et al. (1992), Thorwirth et al. (2000, 2001), Fabricant et al. (1977), Brown et al. (1990), Kryvda et al. (2009), Spezzano et al. (2012), Bizzocchi et al. (2004), and Müller et al. (2005).

${ }^{\mathrm{c}}$ We report the results toward the eight regions described in the text: L1157-1 = L1157-B1 SHOCK 1, L1157-2 = L1157-B1 SHOCK 2, L1157-3 = L1157-B1 SHOCK 3, FIR3 = OMC-2 FIR3, FIR4-W = OMC-2 FIR4 west, FIR4-E = OMC-2 FIR4 east, FIR5 = OMC-2 FIR5, IRAS4Ac = IRAS4A hot corino, and IRAS4As = IRAS4A shocked region. 


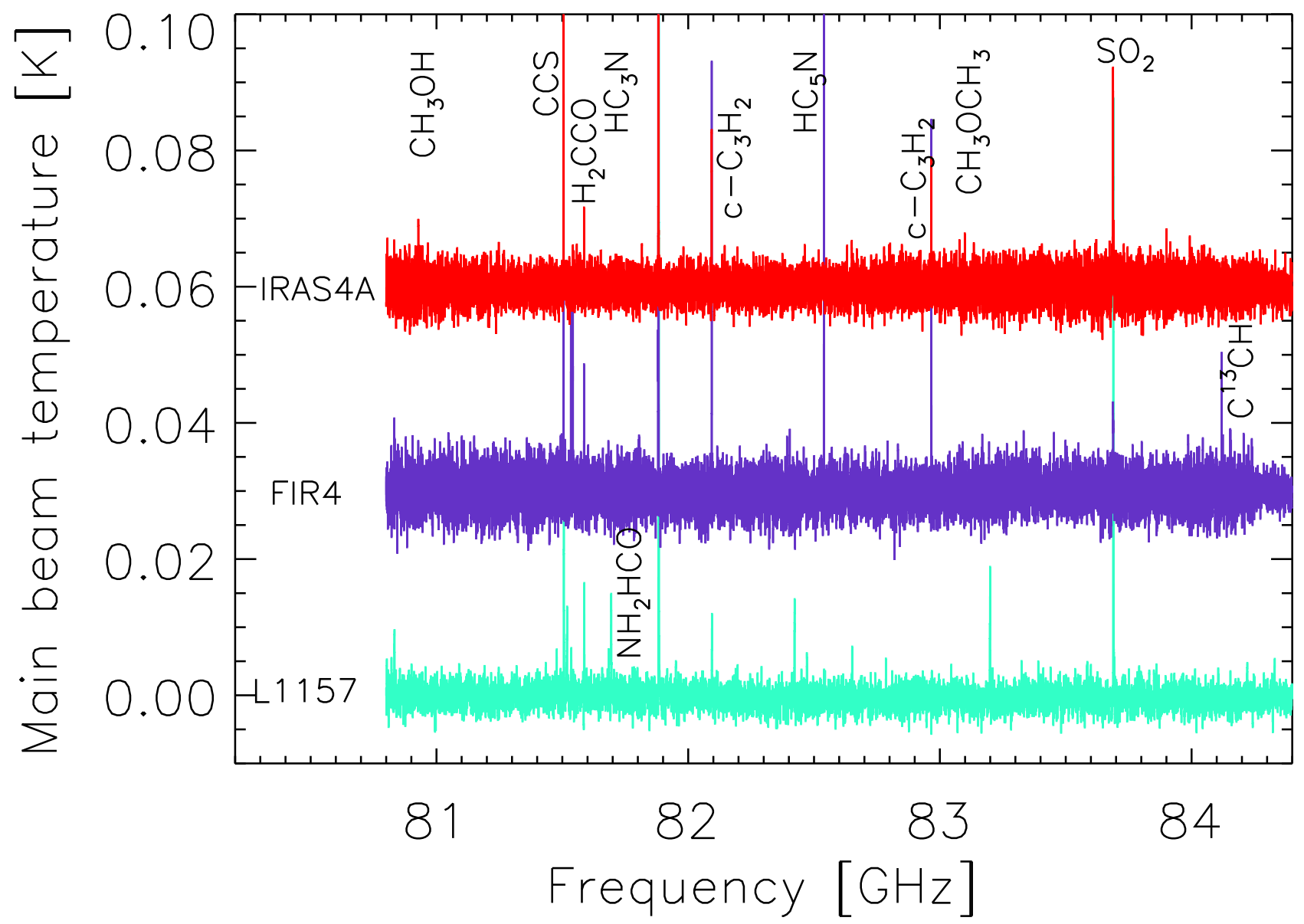

Figure 4. IRAM-30 m spectra of the three regions L1157-B1, OMC-2 FIR4, and IRAS4A in the same frequency range of Figure 2. The integrated intensity is in main beam temperature $(\mathrm{K})$ and the frequency in $\mathrm{GHz}$. The brightest detected lines are labeled at the top and bottom of the plot. The data were taken in the framework of the Large Project ASAI (Lefloch et al. 2017a).

Table 6

Integrated Intensity in $\mathrm{K} \mathrm{km} \mathrm{s}^{-1}$ of the Detected Lines Listed in Table 5

\begin{tabular}{|c|c|c|c|c|c|c|c|c|c|c|}
\hline \multirow{2}{*}{ Species } & \multirow{2}{*}{$\begin{array}{c}\text { Frequency } \\
\quad(\mathrm{GHz})\end{array}$} & \multicolumn{9}{|c|}{ Integrated Flux $\left(\mathrm{K} \mathrm{km} \mathrm{s}^{-1}\right)$} \\
\hline & & IRAS4As & IRAS4Ac & FIR5 & FIR4-W & FIR4-E & FIR3 & L1157-1 & L1157-2 & L1157-3 \\
\hline $\mathrm{CH}_{3} \mathrm{OH}$ & 80.993 & $\cdots$ & 0.195 & $\cdots$ & $\cdots$ & 0.305 & $\cdots$ & $\cdots$ & $\cdots$ & $\cdots$ \\
\hline $\mathrm{CCS}$ & 81.505 & $\cdots$ & $\ldots$ & $\cdots$ & $\ldots$ & 0.172 & $\ldots$ & 0.132 & 0.139 & $\cdots$ \\
\hline $\mathrm{HC}^{13} \mathrm{CCN}$ & 81.534 & $\ldots$ & $\ldots$ & $\ldots$ & $\ldots$ & 0.081 & $\ldots$ & $\ldots$ & 0.045 & $\ldots$ \\
\hline $\mathrm{HCC}^{13} \mathrm{CN}$ & 81.542 & $\ldots$ & $\ldots$ & $\cdots$ & $\ldots$ & 0.079 & $\cdots$ & $\cdots$ & 0.046 & $\cdots$ \\
\hline $\mathrm{H}_{2} \mathrm{CCO}$ & 81.586 & 0.042 & 0.082 & $\cdots$ & 0.077 & 0.113 & $\ldots$ & $\ldots$ & 0.135 & $\cdots$ \\
\hline $\mathrm{NH}_{2} \mathrm{CHO}$ & 81.693 & 0.041 & 0.058 & & 0.039 & 0.053 & $\cdots$ & $\ldots$ & 0.086 & 0.074 \\
\hline $\mathrm{HC}_{3} \mathrm{~N}$ & 81.881 & 0.091 & 0.309 & 0.524 & 2.039 & 4.202 & 0.360 & 2.116 & 3.751 & 1.565 \\
\hline $\mathrm{c}-\mathrm{C}_{3} \mathrm{H}_{2}$ & 82.093 & $\ldots$ & $\ldots$ & $\ldots$ & 0.080 & 0.102 & $\ldots$ & 0.075 & 0.057 & $\ldots$ \\
\hline $\mathrm{HC}_{5} \mathrm{~N}$ & 82.539 & $\cdots$ & $\ldots$ & 0.063 & $\ldots$ & 0.177 & $\cdots$ & $\ldots$ & $\ldots$ & \\
\hline $\mathrm{c}-\mathrm{C}_{3} \mathrm{H}_{2}$ & 82.966 & $\ldots$ & 0.070 & $\ldots$ & 0.078 & 0.135 & $\ldots$ & $\ldots$ & $\ldots$ & $\ldots$ \\
\hline $\mathrm{SO}_{2}$ & 83.688 & 0.061 & 0.385 & $\ldots$ & 0.378 & 0.192 & 0.093 & 0.331 & 1.020 & 0.533 \\
\hline $\operatorname{rms}(\mathrm{K})$ & $\ldots$ & 0.001 & 0.002 & 0.001 & 0.010 & 0.005 & 0.001 & 0.005 & 0.008 & 0.008 \\
\hline
\end{tabular}

Note. The last row reports the rms in $\mathrm{K}$ toward each region.

$\left(8_{1,7}-8_{0,8}\right)$ line ratio of $3-6$. This is indeed consistent with previous observations that show an enhancement in shocked regions of $\mathrm{SO}_{2}$ but not of $\mathrm{HC}_{3} \mathrm{~N}$ (e.g., Bachiller \& Pérez Gutiérrez 1997; Benedettini et al. 2013).

Even more marked is the difference in intensity of weaker lines. Interestingly, the CCS $\left(6_{7}-5_{6}\right)$ line is relatively bright in
L1157-B1 SHOCKS 1 and 2, and in OMC-2 FIR4-E, whereas it is undetected in the other sources/regions. On the contrary, the $\mathrm{H}_{2} \mathrm{CCO}\left(4_{1,3}-3_{1,2}\right)$ line is bright in L1157-B1 SHOCK2 and OMC-2 FIR4-E, weak in NGC 1333-IRAS4Ac and OMC-2 FIR4-W, and undetected elsewhere. Also, in this case, the largest differences are likely caused by a real difference in 


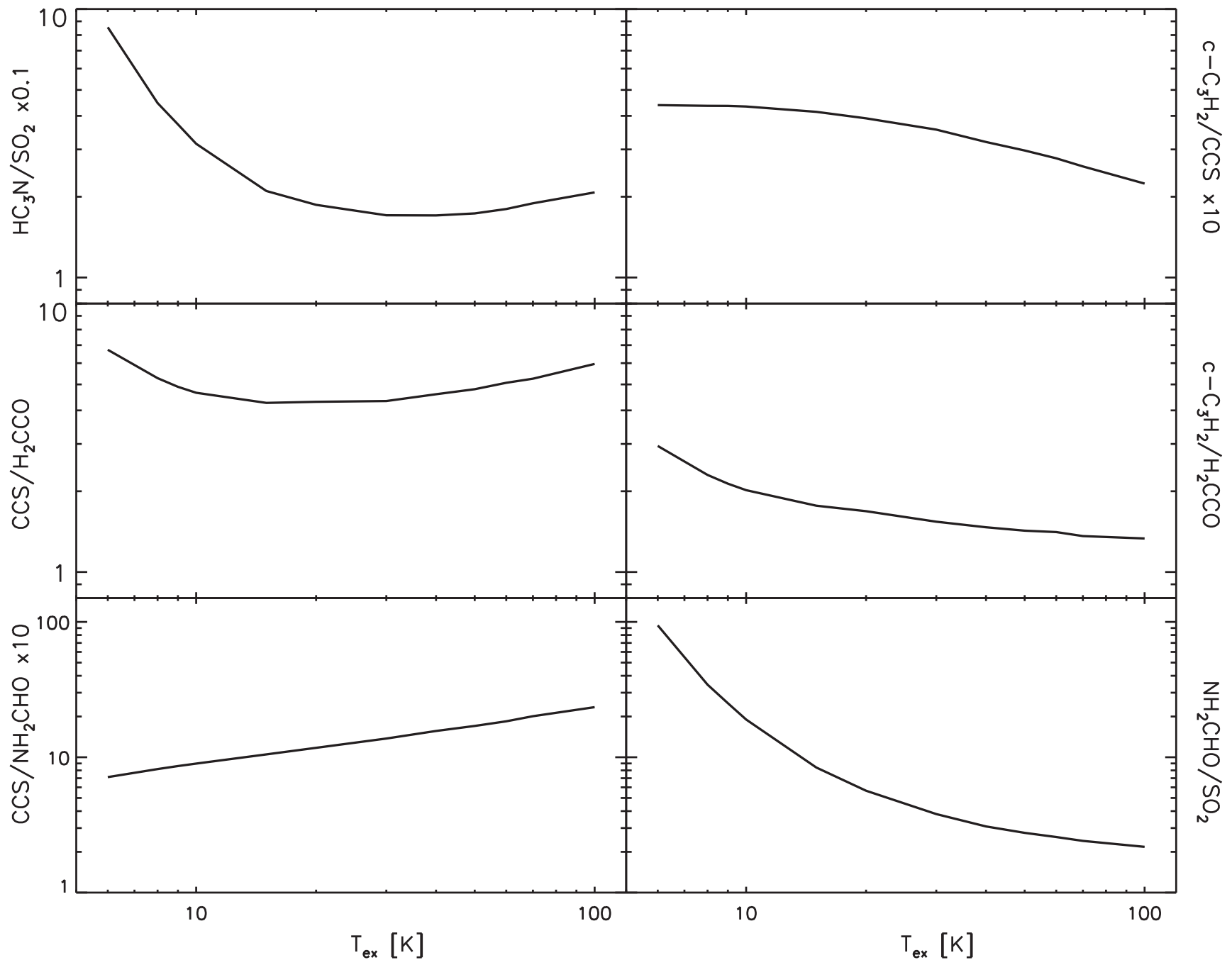

Figure 5. Line ratios as a function of the excitation temperature $T_{\mathrm{ex}}$ : $\mathrm{HC}_{3} \mathrm{~N} 9-8 / \mathrm{SO}_{2} 8_{1,7}-8_{0,8}$, multiplied by a factor 0.1 (left top), $\mathrm{CCS} 6_{7}-5_{6} / \mathrm{H}_{2} \mathrm{CCO} 4_{1,3}-3_{1,2}$ (left middle), CCS $6_{7}-5_{6} / \mathrm{NH}_{2} \mathrm{CHO} 4_{1,4}-3_{1,3}$ (left bottom), c- $\mathrm{C}_{3} \mathrm{H}_{2} 3_{1,2}-3_{0,3} / \mathrm{CCS} 6_{7}-5_{6}$ multiplied by a factor 10 (right top), c-C $\mathrm{C}_{3} \mathrm{H}_{2} 3_{1,2}-3_{0,3} / \mathrm{H}_{2} \mathrm{CCO} 4_{1,3}-3_{1,2}$ (right middle), and $\mathrm{NH}_{2} \mathrm{CHO} 4_{1,4}-3_{1,3} / \mathrm{SO}_{2} 8_{1,7}-8_{0,8}$ (right bottom). The line ratios were computed assuming the same column density for all species, LTE populated and optically thin lines.

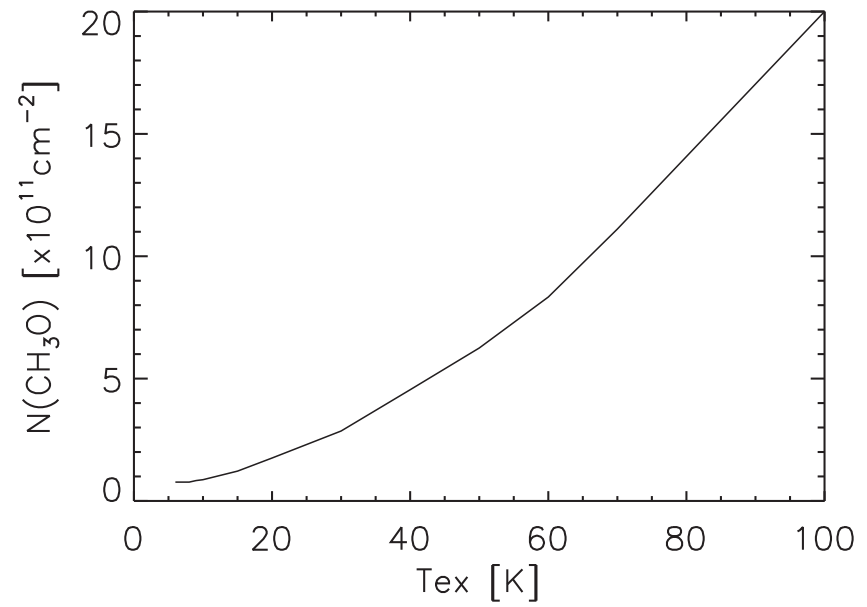

Figure 6. Upper limit to the methoxy column density as a function of the excitation temperature, derived assuming a rms of $1 \mathrm{mK}$, a line width of $2 \mathrm{~km} \mathrm{~s}^{-1}$.

chemical composition rather than excitation conditions, as the two lines have, again, similar upper level energies. This is quantitatively shown in Figure 5: the CCS $\left(6_{7}-5_{6}\right)$ over $\mathrm{H}_{2} \mathrm{CCO}$ $\left(4_{1,3}-3_{1,2}\right)$ line ratio is practically constant for an excitation temperature, varying between 6 and $100 \mathrm{~K}$.

More relevant for the present work and the goal of SOLIS, lines from formamide are variably present in the sources/ regions. For example, the $\mathrm{NH}_{2} \mathrm{CHO}\left(4_{1,4}-3_{1,3}\right)$ line is detected in L1157-B1 SHOCKS 2 and 3, NGC 1333-IRAS4A hot corino and shock, tentatively in OMC-2 FIR4 west and east, and undetected in OMC-2 FIR3 and FIR5 (Figure 3). When considering the ratio between the formamide and CCS lines, which have very similar upper level energies and Einstein coefficients, the chemical differentiation is particularly evident with $\mathrm{CCS} / \mathrm{NH}_{2} \mathrm{CHO}$ varying from 1.5 to 3 in OMC-2 FIR4-E and L1157-B1 SHOCK 2 to less than unity in the other regions where $\mathrm{NH}_{2} \mathrm{CHO}$ is detected. Figure 5 quantifies how different the excitation conditions should be to explain the observed difference in the CCS $/ \mathrm{NH}_{2} \mathrm{CHO}$ line ratio. For excitation temperatures between 10 and $80 \mathrm{~K}$, the line ratio varies by no more than a factor of two. Therefore, dedicated studies of the various sources/regions, coupled with chemical modeling, as in Codella et al. (2017), are needed to shed light on the processes behind the observed line intensity differences among the various sources. 
In summary, the distribution and relative abundances of (complex) organic molecules change significantly among the different sources, and this diversity is already clear at 300-3000 au scales.

\subsection{Differences within the Sources}

\subsubsection{NGC 1333-IRAS4A}

The spectra of the two studied regions, the hot corino and the shocked region, are definitively very different (Figure 1). The hot corino shows the typical lines of iCOMs (not reported in Tables 5 and 6 because they are many but not relevant in the context of this article: the entire list will be the subject of a forthcoming publication), such as formamide, dimethyl ether, and methanol, whereas the shocked region possesses only two (relatively) bright lines characteristic of hot corinos, the $\mathrm{NH}_{2} \mathrm{CHO}\left(4_{1,4}-3_{1,3}\right)$ and $\mathrm{SO}_{2}\left(8_{1,7}-8_{0,8}\right)$ lines. The distribution of the $\mathrm{SO}_{2}$ line intensity is shown in Figure 1. The line clearly maps the contour of the outflowing/shocked gas. Particularly intriguing is the formamide detection toward the shocked region, in a position that is at the border of the region where $\mathrm{SO}_{2}$ emits. Note that $\mathrm{SO}_{2}$ traces fairly well the two jets emitted by $\mathrm{A} 1$ and A2, observed in SiO by Santangelo et al. (2015) and here unresolved. As in the case of L1157-B1 (Codella et al. 2017; S. Feng et al. 2017, in preparation), the $\mathrm{SO}_{2}$ versus formamide spatial segregation could be caused by a time effect, in addition to the release in the gas phase of the formamide precursors, namely formaldehyde and amidogen. It is worth mentioning that methanol is, rather surprisingly, not detected toward the shocked region, where formamide is present (Table 6). In addition, the shocked region emits weak lines of $\mathrm{H}_{2} \mathrm{CCO}$ and $\mathrm{HC}_{3} \mathrm{~N}$ (9-8), also detected in the hot corino. A forthcoming article will report on a detailed analysis of this jet plus shocked region (A. LópezSepulcre et al. 2017, in preparation).

\subsubsection{OMC-2 FIR4}

The three objects in the NOEMA primary beam, FIR3, FIR4 and FIR5, show clear and remarkable differences. The difference in the cyanopolyynes and $\mathrm{c}-\mathrm{C}_{3} \mathrm{H}_{2}$ are discussed in Fontani et al. (2017) and Favre et al. (2017), respectively. Briefly, cyanopolyynes probe the presence of a source of energetic $(\geqslant 10 \mathrm{Mev})$ particles irradiating the eastern part of OMC-2 FIR4 and FIR5. c- $\mathrm{C}_{3} \mathrm{H}_{2}$ is, instead, a good thermometer of the region, which shows a rather uniform temperature with a possible small increasing gradient toward east, as also found with the low angular resolution temperature map from $\mathrm{NH}_{3}$ (Friesen et al. 2017). This is also consistent with the fact that the region is irradiated by energetic particles at/from the east.

In addition, the spectra in Figures 2 and 3 indicate the presence of a high lying $\left(E_{\mathrm{up}}=103 \mathrm{~K}\right)$ line of $\mathrm{CH}_{3} \mathrm{OH}$, typical of hot corinos, only toward FIR4-E. The $\mathrm{SO}_{2}$ line is bright toward FIR4-W and FIR4-E, and it is detected in FIR3 but not in FIR5 (Table 6). Since $\mathrm{SO}_{2}$ is often also a hot corino species or, more generally, a warm gas indicator, this would suggest that FIR3 harbors a young protostar, maybe a hot corino, while FIR5 is a cold object. Similarly, one or more hot corinos might be present in the FIR4 region. Toward FIR4, the eastern part (the one irradiated by energetic particles) is the region with the richest spectrum, with all of the lines listed in Table 6 detected. It is likely that one or more hot cores/corinos are embedded, therefore, in the east region of FIR4. In the western part, only $\mathrm{H}_{2} \mathrm{CCO}$ and c- $\mathrm{C}_{3} \mathrm{H}_{2}$ are detected. In general, they are species that are abundant in the exterior of molecular clouds, where residual UV photons produce a (small) fraction of neutral carbon (e.g., Spezzano et al. 2016).

We do not find any evidence that FIR4 north is affected by the outflow from FIR3, as suggested by Shimajiri et al. (2015). An increase of the gas temperature or broadband emission at north are also not detected in the SOLIS observations. Since the Shimajiri et al. observations were carried out with single-dish telescopes with a beam of $\sim 20^{\prime \prime}$, it is possible that the emission that they observed is contaminated by the hot corinos in the east FIR4 region.

Finally, a weak formamide line is present in both FIR4-E and FIR4-W. To confirm its detection, additional SOLIS observations are underway. Finally, incoming higher angular resolution SOLIS observations will definitively establish whether hot corinos or shocks or both are present and, if so, where in the region (R. Neri et al. 2017, in preparation).

\subsection{3. $L 1157-B 1$}

L1157-B1 can be separated into three zones when the relative abundance of acetaldehyde and formamide is considered (Codella et al. 2017). The three zones identify three different shocks caused by the episodic ejections and the resulting jet impacting the cavity wall excavated by the outflow from the central star. The three shocks have been created at different times, within a lapse of time of about $1000 \mathrm{yr}$ and with the one in the north being the youngest one (Podio et al. 2016). The difference between the three zones is also evident looking at the $\mathrm{CCS}, \mathrm{H}_{2} \mathrm{CCO}$ and $\mathrm{c}-\mathrm{C}_{3} \mathrm{H}_{2}$ lines. The $\mathrm{CCS}$ and $\mathrm{c}-\mathrm{C}_{3} \mathrm{H}_{2}$ lines are bright in the north and central zones, but absent in the south, whereas $\mathrm{H}_{2} \mathrm{CCO}$ is only bright in the central zone. This diversity in the CCS, c- $\mathrm{C}_{3} \mathrm{H}_{2}$, and $\mathrm{H}_{2} \mathrm{CCO}$ line distribution cannot be attributed to sensitivity, but must reflect a difference in the excitation conditions or chemical composition of the three regions. The upper level energies of the CCS, $\mathrm{H}_{2} \mathrm{CCO}$, and $\mathrm{c}-\mathrm{C}_{3} \mathrm{H}_{2}\left(3_{1,2}-3_{0,3}\right)$ transitions are 15,22 , and $16 \mathrm{~K}$, whereas the Einstein coefficients are 2.5, 0.5, and $1.0 \times 10^{-5} \mathrm{~s}^{-1}$, respectively, so that different excitation conditions seem unlikely. The plots of these line ratios, reported in Figure 5, support this interpretation. Based on the analysis of formamide, the line of which is detected only toward SHOCKs 2 and 3, we suggest that the differentiation of $\mathrm{CCS}, \mathrm{H}_{2} \mathrm{CCO}$ and $\mathrm{c}-\mathrm{C}_{3} \mathrm{H}_{2}$ is also linked to the evolution of the gas chemical composition on a short timescale ( $\sim 1000$ years; Codella et al. 2017).

\subsection{Methoxy}

Setup 1 was selected in order to have lines from formamide and methoxy in the Narrow Band correlator (Table 4). While formamide is detected in all three sources (but not in all regions of each source: Table 5), methoxy is undetected in all of them. The upper limit to the methoxy column density of each source depends on the exact physical conditions. However, to have an order of magnitude, adopting an rms of $1 \mathrm{mK}$ (Table 6) and a line width of $2 \mathrm{~km} \mathrm{~s}^{-1}$, the upper limit to the methoxy column density varies from $8 \times 10^{10}$ to $2 \times 10^{12} \mathrm{~cm}^{-2}$ for an excitation temperature from 6 to $100 \mathrm{~K}$ (Figure 6). ${ }^{26}$ This provides a very approximate upper limit to the methoxy

\footnotetext{
26 The upper limit does not apply to extended emission filtered out by the NOEMA interferometer.
} 
abundance in OMC-2 FIR4 and L1157-B1 of $2 \times 10^{-12}$ and $3 \times 10^{-10}$, assuming an excitation temperature of 30 and $50 \mathrm{~K}$ and a $\mathrm{H}_{2}$ column density of $2 \times 10^{23}$ and $2 \times 10^{21} \mathrm{~cm}^{-2}$ (Lefloch et al. 2012; Fontani et al. 2017), respectively. The upper limit to the methoxy abundance in IRAS4A is approximately $1 \times 10^{-11}$, assuming an excitation temperature of $70 \mathrm{~K}$, a $\mathrm{H}_{2}$ column density of $1 \times 10^{24} \mathrm{~cm}^{-2}$, and a hot corino size of 1"! 2 (López-Sepulcre et al. 2017).

Single-dish observations of cold prestellar objects detected methoxy so far only in a handful of cold prestellar cores (Cernicharo et al. 2012; Bacmann \& Faure 2016; Jiménez-Serra et al. 2016). The measured column densities are about $10^{12} \mathrm{~cm}^{-2}$. The derivation of the abundance from these single-dish observations is rather tricky, as it depends on where the observed methoxy line emission originates (both for the correction of the filling factor and the adopted $\mathrm{H}_{2}$ column density). Vastel et al. (2014) claimed that iCOMs emission arises at the border of the prestellar condensations. JiménezSerra et al. (2016) indeed showed that methoxy is about 10 times more abundant in the border of the prestellar core L1544 than in its interior. Specifically, Jiménez-Serra et al. measured a methoxy abundance equal to $\sim 3 \times 10^{-11}$ and an upper limit of $5 \times 10^{-12}$ at the border and in the interior of L1544, which are relatively well reproduced (within a factor of 3) by Vasyunin et al. (2017). These values are close to the upper limits computed previously from the present SOLIS observations. A forthcoming SOLIS article (F. Dulieu et al. 2017, in preparation) will analyze the chemistry of methoxy using a more appropriate and dedicated modeling of the SOLIS protostellar sources.

\section{Conclusions}

Some interstellar complex organic molecules, in this work called iCOMs, might have had a role in the emergence of life on Earth, constituting the smallest bricks to build up biotic macromolecules. A few tens of iCOMs are detected so far toward one or two massive star-forming regions, SgrB2 and Orion KL. In Solar-like star-forming regions, the number is reduced to a bit more than a dozen, mostly detected in one source, IRAS16293-2422 (see the introduction), and only a few of them in other sources (e.g., López-Sepulcre et al. 2017). Given the paucity of sources where the species are detected, it is difficult to assess how the environment affects the presence and abundance of iCOMs. In turn, this lack of information severely hampers our understanding of how iCOMs form in the ISM. From a theoretical point of view, two major routes of formation are possible: on the grain surfaces or in the gas. More likely, a combination of the two is at work, but the "who does what" is still largely debated.

The advent of new powerful facilities, like IRAM-NOEMA and ALMA, is opening a new era in the study of iCOMs, especially with Solar-like star-forming regions, the ones with the highest potential in terms of molecules having a possible role in the emergence of terrestrial life. The project PILS (Protostellar Interferometric Line Survey: Jørgensen et al. 2016) is already providing a new census of iCOMs toward IRAS16293-2422, which improves the old one by TIMASSS (The IRAS16293-2422 millimeter and submillimeter spectral survey: Caux et al. 2011). In this article, we present a new IRAM-NOEMA Large Program called SOLIS (Seeds Of Life In Space), the goal of which is to obtain the spatial distribution of selected iCOMs in half a dozen Solar-like star-forming regions. The SOLIS images have spatial resolutions of $1^{\prime \prime}-5^{\prime \prime}$, to allow studies on scales of 100-5000 au. This will allow us to put constraints on the formation routes of iCOMs, as well as on the mechanisms that inject them from the grain mantles to the gas, where they are detected, and the role of UV illumination.

Here we present the SOLIS project and a summary of the first results obtained from three sources: NGC 1333-IRAS4A, OMC-2 FIR4, and L1157-B1. They show the potential of obtaining interferometric images of iCOMs. Comparing the SOLIS and ASAI spectra, we can disentangle the molecular lines emitted in the large-scale envelopes and molecular clouds harboring the sources from those in the immediate vicinity of where the action takes place-namely hot corinos and shocks.

Also, the SOLIS small scale spectra show a clear differentiation in the three studied sources, caused by the different physical conditions in the three different environments. Particularly spectacular is the difference in the abundance distribution of two cyanopolyynes, $\mathrm{HC}_{3} \mathrm{~N}$ and $\mathrm{HC}_{5} \mathrm{~N}$. This is likely caused by a difference in the rate of energetic $(\geqslant 10 \mathrm{MeV})$ particles irradiating the various studied sources, as discussed in detail by Fontani et al. (2017). A similar conclusion is reached by Favre et al. (2017) from the analysis of the $\mathrm{c}-\mathrm{C}_{3} \mathrm{H}_{2}$ emission.

In the setup reported in this study, the SOLIS target iCOM is formamide. It is firmly detected in the hot corino of NGC 1333-IRAS4A, and, for the first time, in the shocked regions of L1157-B1 and NGC 1333-IRAS4A, created by their respective violent ejections of material. In OMC-2 FIR4, there is a tentative detection, which needs to be confirmed by forthcoming SOLIS formamide observations. The analysis of the distribution of the formamide abundance provides strong constraints on the formation route of this species. This is discussed in detail in Codella et al. (2017), who concluded that the gas-phase reaction proposed by Kahane et al. (2013) and studied by Barone et al. (2015), Vazart et al. (2016), and Skouteris et al. (2017) reproduces the SOLIS observations very well.

The other molecule targeted in the setup presented here is methoxy. This molecule is not detected in any of the three sources, leading to upper limits to its abundance of $\sim 10^{-12}$ $-10^{-11}$, namely the same order of magnitude of that measured in cold prestellar cores (e.g., Jiménez-Serra et al. 2016). A forthcoming article will exploit this information in detail to set constraints on the methoxy chemistry.

Further studies based on SOLIS, as the ones mentioned here, will put additional strong constraints on the formation and destruction routes of formamide and, hopefully, also more iCOMs, particularly those targeted by SOLIS.

We acknowledge the funding from the European Research Council (ERC), projects PALs (contract 320620) and DOC (contract 741002). This work was supported by the French program Physique et Chimie du Milieu Interstellaire (PCMI), funded by the Conseil National de la Recherche Scientifique (CNRS) and Centre National dEtudes Spatiales (CNES) and by the Italian Ministero dell'Istruzione, Universitá e Ricerca, through the grant Progetti Premiali 2012-iALMA (CUP C52I13000140001). Partial salary support for A. Pon was provided by a Canadian Institute for Theoretical Astrophysics (CITA) National Fellowship. I.J.-S. and D.Q. acknowledge the financial support received from the STFC through an Ernest 
Rutherford Fellowship and Grant (proposal numbers ST/ L004801 and ST/M004139).

\section{ORCID iDs}

C. Ceccarelli (ㄷ) https://orcid.org/0000-0001-9664-6292

P. Caselli (i) https://orcid.org/0000-0003-1481-7911

F. Fontani (i) https://orcid.org/0000-0003-0348-3418

R. Neri (i) https://orcid.org/0000-0002-7176-4046

C. Codella (1) https://orcid.org/0000-0003-1514-3074

J. E. Pineda (1i) https://orcid.org/0000-0002-3972-1978

F. Alves $(1)$ https://orcid.org/0000-0002-7945-064X

R. Bachiller (10) https://orcid.org/0000-0002-5331-5386

N. Balucani (1) https://orcid.org/0000-0001-5121-5683

L. Bizzocchi (1) https://orcid.org/0000-0002-9953-8593

P. Hily-Blant (1) https://orcid.org/0000-0003-3488-8442

J. Holdship (1) https://orcid.org/0000-0003-4025-1552

J. Laas (i) https://orcid.org/0000-0001-6876-6940

Y. Oya (ib https://orcid.org/0000-0002-0197-8751

A. Pon (i) https://orcid.org/0000-0003-4612-1812

A. Punanova (i) https://orcid.org/0000-0001-6004-875X

A. Rimola (i) https://orcid.org/0000-0002-9637-4554

N. Sakai (i) https://orcid.org/0000-0002-3297-4497

V. Taquet (ib https://orcid.org/0000-0003-0407-7489

L. Testi (1) https://orcid.org/0000-0003-1859-3070

A. I. Vasyunin (1) https://orcid.org/0000-0003-1684-3355

S. Viti (1) https://orcid.org/0000-0001-8504-8844

\section{References}

Adams, F. C. 2010, ARA\&A, 48, 47

Altwegg, K., Balsiger, H., Bar-Nun, A., et al. 2016, SciA, 2, 1600285

Arce, H. G., Santiago-García, J., Jørgensen, J. K., Tafalla, M., \& Bachiller, R. 2008, ApJL, 681, L21

Bachiller, R., Guilloteau, S., Gueth, F., et al. 1998, A\&A, 339, L49

Bachiller, R., \& Pérez Gutiérrez, M. 1997, ApJL, 487, L93

Bachiller, R., Peréz Gutiérrez, M., Kumar, M. S. N., \& Tafalla, M. 2001, A\&A, 372,899

Bacmann, A., \& Faure, A. 2016, A\&A, 587, 130

Bacmann, A., Lefloch, B., Ceccarelli, C., et al. 2003, ApJL, 585, L55

Bacmann, A., Taquet, V., Faure, A., Kahane, C., \& Ceccarelli, C. 2012, A\&A, 541,12

Balucani, N., Ceccarelli, C., \& Taquet, V. 2015, MNRAS, 449, L16

Barone, V., Latouche, C., Skouteris, D., et al. 2015, MNRAS, 453, L31

Belloche, A., Meshcheryakov, A. A., Garrod, R. T., et al. 2017, A\&A, 601, 49

Benedettini, M., Viti, S., Codella, C., et al. 2013, MNRAS, 436, 179

Bennett, C., \& Kaiser, R. I. 2007, ApJ, 661, 899

Bianchi, E., Codella, C., Ceccarelli, C., et al. 2017, MNRAS, 467, 3011

Bisschop, S. E., Jørgensen, J. K., Bourke, T. L., Bottinelli, S., \& van Dishoeck, E. F. 2008, A\&A, 488, 959

Bizzocchi, L., Caselli, P., Spezzano, S., \& Leonardo, E. 2014, A\&A, 569, 27

Bizzocchi, L., Degli Esposti, C., \& Botschwina, P. 2004, JMoSp, 225, 145

Bottinelli, S., Ceccarelli, C., Lefloch, B., et al. 2004, ApJ, 615, 354

Bourke, T. L., Myers, P. C., Evans, N. J., II, et al. 2006, ApJL, 649, L37

Brown, R. D., Godfrey, P. D., McNaughton, D., Pierlot, A. P., \& Taylor, W. H. 1990, JMoSp, 140, 340

Caselli, P., \& Ceccarelli, C. 2012, A\&ARv, 20, 56

Caselli, P., Keto, E., Bergin, E. A., et al. 2012, ApJL, 759, L37

Caselli, P., van der Tak., F. F. S., Ceccarelli, C., \& Bacmann, A. 2003, A\&A, 403, L37

Caselli, P., Walmsley, C. M., Tafalla, M., et al. 1999, ApJL, 523, L165

Caselli, P., Walmsley, C. M., Zucconi, A., et al. 2002, ApJ, 565, 344

Caux, E., Kahane, C., Castets, A., et al. 2011, A\&A, 532, 23

Cazaux, S., Tielens, A. G. G. M., Ceccarelli, C., Castets, A., Wakelam, V., et al. 2003, ApJL, 593, L51

Ceccarelli, C., Bacmann, A., Boogert, A., et al. 2010, A\&A, 521, L22

Ceccarelli, C., Caselli, P., Herbst, E., Tielens, A. G. G. M., \& Caux, E. 2007, in Protostars and Planets V, ed. B. Reipurth, D. Jewitt, \& K. Keil (Tucson, AZ: Univ. Arizona Press), 47

Ceccarelli, C., Castets, A., Caux, E., et al. 2000a, A\&A, 355, 1129
Ceccarelli, C., Dominik, C., López-Sepulcre, A., et al. 2014, ApJL, 790, 1 Ceccarelli, C., Loinard, L., Castets, A., Faure, A., \& Lefloch, B. 2000b, A\&A, 362,1122

Cernicharo, J., Marcelino, N., Roueff, E., et al. 2012, ApJ, 759, 43

Chen, X., Launhardt, R., \& Henning, Th. 2009, ApJ, 691, 1729

Chini, R., Reipurth, B., Sievers, A., et al. 1997, A\&A, 325, 542

Codella, C., Bachiller, R., \& Reipurth, B. 1999, A\&A, 343, 585

Codella, C., Cabrit, S., Gueth, F., et al. 2007, A\&A, 462, L53

Codella, C., Ceccarelli, C., Bianchi, E., et al. 2016, MNRAS, 462, L75

Codella, C., Ceccarelli, C., Caselli, P., et al. 2017, A\&A, 605, L3

Codella, C., Fontani, F., Ceccarelli, C., et al. 2015, MNRAS, 449, L11

Coutens, A., Jørgensen, J. K., Wampfler, S. F., \& Lykke, J. M. 2015, A\&A, 576,5

Crapsi, A., Caselli, P., Walmsley, C. M., \& Tafalla, M. 2007, A\&A, 470, 221

Crapsi, A., Caselli, P., Walmsley, M., et al. 2004, A\&A, 420, 957

Crimier, N., Ceccarelli, C., Lefloch, B., \& Faure, A. 2009, A\&A, 506, 1229

De Duve, C. 2005, Singularities: Landmarks on the Patways of Life (Cambridge: Cambridge Univ. Press)

De Duve, C. 2011, RSPTA, 369, 620

De Simone, M., Codella, C., Testi, L., et al. 2017, A\&A, 599, 121

Dunham, M. M., Evans, N. J., II, Bourke, T. J., et al. 2006, ApJ, 651, 945

Eislöffel, J., Smith, M. D., Davis, C. J., \& Ray, T. P. 1996, AJ, 112, 2086

Elsila, J. E., Glavin, D. P., \& Dworkin, J. P. 2009, M\&PS, 44, 1323

Enrique-Romero, J., Rimola, A., Ceccarelli, C., \& Balucani, N. 2016, MNRAS, 459, L6

Fabricant, B., Krieger, D., \& Muenter, J. S. 1977, JChPh, 67, 1576

Favre, C., Ceccarelli, C., López-Sepulcre, A., et al. 2017, ApJ, submitted

Fedoseev, G., Chuang, K.-J., van Dishoeck, E. F., Ioppolo, S., \& Linnartz, H. 2016, MNRAS, 460, 4297

Few, R. W., \& Cohen, R. J. 1983, MNRAS, 203, 853

Fontani, F., Ceccarelli, C., Favre, C., et al. 2017, A\&A, 605, 57

Fontani, F., Codella, C., Ceccarelli, C., et al. 2014, ApJL, 788, L43

Friesen, R. K., Pineda, J., Rosolowsky, E., et al. 2017, ApJ, 843, 63

Furlan, E., Megeath, S. T., Osorio, M., et al. 2014, ApJ, 786, 26

Garrod, R. T., \& Herbst, E. 2006, A\&A, 457, 927

Garrod, R. T., Widicus Weaver, S. L., \& Herbst, E. 2008, ApJ, 682, 283

Gonzalez-Garcia, B., Manoj, P., Watson, D. M., et al. 2016, A\&A, 596, 26

Gounelle, M., Shu, F. H., Shang, H., et al. 2006, ApJ, 640, 1163

Gueth, F., Guilloteau, S., \& Bachiller, R. 1996, A\&A, 307, 891

Gueth, F., Guilloteau, S., \& Bachiller, R. 1998, A\&A, 333, 287

Herbst, E., \& van Dishoeck, E. F. 2009, ARA\&A, 47, 427

Hirota, T., Bushimata, T., Choi, Y. K., et al. 2007, PASJ, 59, 89

Hirota, T., Bushimata, T., Choi, Y. K., et al. 2008, PASJ, 60, 37

Jaber, A., Ceccarelli, C., Kahane, C., \& Caux, E. 2014, ApJ, 791, 29

Jaber Al-Edhari, A., Ceccarelli, C., Kahane, C., et al. 2017, A\&A, 597, 40

Jiménez-Serra, I., Vasyunin, A., Caselli, P., et al. 2016, ApJL, 830, L6

Jones, B. M., Bennett, C. J., \& Kaiser, R. I. 2011, ApJ, 734, 78

Jørgensen, J. K., Favre, C., Bisschop, S., et al. 2012, ApJL, 757, L4

Jørgensen, J. K., van der Wiel, M. H., Coutens, A., et al. 2016, A\&A, 595, 117

Kahane, C., Ceccarelli, C., Faure, A., \& Caux, E. 2013, ApJL, 763, L38 Kanuchova, G., Urso, R., Baratta, G., et al. 2016, A\&A, 585, 155

Karska, A., Herczeg, G. J., \& van Dishoeck, E. F. 2013, A\&A, 552, A141

Kauffmann, J., Bertoldi, F., Evans, N. J., II, et al. 2005, AN, 326, 878

Kenyon, S. J., Dobrzycka, D., \& Hartmann, L. 1994, AJ, 108, 1872

Keto, E., Caselli, P., \& Rawlings, J. 2015, MNRAS, 446, 3731

Kristensen, L. E., van Dishoeck, E. F., Bergin, E. A., Visser, R., \& Yildı, U. A. 2012, A\&A, 542, A8

Kryvda, A. V., Gerasimov, V. G., Dyubko, S. F., Alekseev, E. A., \& Motiyenko, R. A. 2009, JMoSp, 254, 28

Ladd, E. F., \& Hodapp, K.-W. 1997, ApJ, 474, 749

Lefloch, B., Bachiller, R., Ceccarelli, C., et al. 2017b, MNRAS, submitted

Lefloch, B., Cabrit, S., Busquet, G., et al. 2012, ApJL, 757, L25

Lefloch, B., Castets, A., Cernicharo, J., et al. 1998, A\&A, 334, 269

Lefloch, B., Ceccarelli, C., Codella, C., et al. 2017a, MNRAS, 469, L73

Lefloch, B., Eisloeffel, J., \& Lazareff, B. 1996, A\&A, 313, L17

Lefloch, B., Gusdorf, A., Codella, C., et al. 2015, A\&A, 581, 4

Ligterink, N. F. W., Coutens, A., Kofman, V., et al. 2017, MNRAS, 469, 2219

Looney, L. W., Mundy, L. G., \& Welch, W. J. 2000, ApJ, 529, 477

Looney, L. W., Tobin, J. J., \& Kwon, W. 2007, ApJL, 670, L131

López-Sepulcre, A., Jaber, A. A., Mendoza, E., et al. 2015, MNRAS, 449,2438

López-Sepulcre, A., Sakai, N., Neri, R., et al. 2017, A\&A, 606, 121

López-Sepulcre, A., Taquet, V., Sánchez-Monge, Á, et al. 2013, A\&A, 556, 62

Lovas, F. J., Suenram, R. D., Ogata, T., \& Yamamoto, S. 1992, ApJ, 399, 325

Lykke, J. M., Coutens, A., Jørgensen, J. K., et al. 2017, A\&A, 597, 53 
Majumdar, L., Gratier, P., Vidal, T., et al. 2016, MNRAS, 458, 1859

Maret, S., Ceccarelli, C., Tielens, X., et al. 2005, A\&A, 442, 527

Martín-Doménech, R., Rivilla, V. M., Jiménez-Serra, I., et al. 2017, MNRAS, 469, 2230

Matsumoto, T., Onishi, T., Tokuda, K., \& Inutsuka, S. 2015, MNRAS, 449, L123

Maury, A., Belloche, A., André, P., et al. 2014, A\&A, 563, L2

Mendoza, E., Lefloch, B., López-Sepulcre, A., et al. 2014, MNRAS, 445, 15

Mezger, P. G., Zylka, R., \& Wink, J. E. 1990, A\&A, 228, 95

Minissale, M., Dulieu, F., Cazaux, S., \& Hocuk, S. 2016, A\&A, 585, 24

Müller, H. S. P., Schlöder, F., Stutzki, J., \& Winnewisser, G. 2005, JMoSt, 742,215

Müller, H. S. P., Thorwirth, S., Roth, D. A., et al. 2001, A\&A, 370, L49

Müller, S., Beelen, A., Black, J. H., et al. 2013, A\&A, 551, 109

Öberg, K. I., Bottinelli, S., Jørgensen, J. K., \& van Dishoeck, E. F. 2010, ApJ, 716,825

Pineda, J. E., Maury, A. J., Fuller, G. A., et al. 2012, A\&A, 544, L7

Pizzarello, S., Krishnamurthy, R. V., Epstein, S., \& Cronin, J. R. 1991, GeCoA, 55, 905

Podio, L., Codella, C., Gueth, F., et al. 2016, A\&A, 593, L4

Podio, L., Codella, C., Lefloch, B., et al. 2017, MNRAS, 470, L16

Punanova, A., Caselli, P., Chacón-Tanarro, A., et al. 2017, A\&A, submitted

Quenard, D., Taquet, V., Vastel, C., Caselli, P., \& Ceccarelli, C. 2016, A\&A, 585,36

Reipurth, B., Chini, R., Krugel, E., Kreysa, E., \& Sievers, A. 1993, A\&A, 273, 221

Requena-Torres, M. A., Marcelino, N., Jiménez-Serra, I., et al. 2007, ApJL, 655, L37

Requena-Torres, M. A., Martín-Pintado, J., Rodríguez-Franco, A., et al. 2006, A\&A, 455, 971

Rubin, R. H., Swenson, G. W., Jr., Benson, R. C., Tigelaar, H. L., \& Flygare, W. H. 1971, ApJL, 169, L39
Saito, S., Kawaguchi, K., Yamamoto, S., et al. 1987, ApJL, 317, L115

Saladino, R., Botta, G., Pino, S., Costanzo, G., \& Di Mauro, E. 2012, Chem Soc. Rev., 41, 5526

Santangelo, G., Codella, C., Cabrit, S., et al. 2015, A\&A, 584, 126

Sargent, A. 1977, ApJ, 218, 736

Schlafly, E. F., Green, G., Finkbeiner, D. P., et al. 2014, ApJ, 789, 15

Shimajiri, Y., Sakai, T., Kitamura, Y., et al. 2015, ApJS, 221, 31

Shimajiri, Y., Sakai, T., Takahashi, S., Takakuwa, S., et al. 2008, ApJ, 683,255

Skouteris, D., Vazart, F., Ceccarelli, C., et al. 2017, MNRAS, 468, L1

Spezzano, S., Bizzocchi, L., Caselli, P., Harju, J., \& Brünken, S. 2016, A\&A, 592, L11

Spezzano, S., Caselli, P., Bizzocchi, L., Giuliano, B. M., \& Lattanzi, V. 2017, A\&A, 606, 82

Spezzano, S., Tamassia, F., Thorwirth, S., et al. 2012, ApJS, 200, 1

Taquet, V., López-Sepulcre, A., Ceccarelli, C., et al. 2015, ApJ, 804, 81

Thorwirth, S., Müller, H. S. P., \& Winnewisser, G. 2000, JMoSp, 204, 133

Thorwirth, S., Müller, H. S. P., \& Winnewisser, G. 2001, PCCP, 3, 1236

Tobin, J. J., Hartmann, L., \& Loinard, L. 2010, ApJL, 722, L12

Tobin, J. J., Looney, L. W., Li, Z.-Y., et al. 2016, ApJ, 818, 73

Tokuda, K., Onishi, T., Matsumoto, T., et al. 2016, ApJ, 826, 26

Tokuda, K., Onishi, T., Saigo, K., et al. 2014, ApJL, 789, L4

van Dishoeck, E. F., Blake, G. A., Jansen, D. J., \& Groesbeck, T. D. 1995 ApJ, 447, 760

Vastel, C., Caselli, P., Ceccarelli, C., et al. 2006, ApJ, 645, 1198

Vastel, C., Ceccarelli, C., Lefloch, B., \& Bachiller, R. 2014, ApJL, 795, L2

Vasyunin, A., \& Herbst, E. 2013, ApJ, 769, 34

Vasyunin, A. I., Caselli, P., Dulieu, F., \& Jiménez-Serra, I. 2017, ApJ, 842, 33

Vazart, F., Calderini, D., Puzzarini, C., et al. 2016, J. Chem. Theory Comput., 12,5385

Xu, L.-H., \& Lovas, F. J. 1997, JPCRD, 26, 17 